\title{
Biopesticidal Potential of Nerolidol, a Sesquiterpene Compound, and its Drastic Impact on Growth and Metamorphosis of the Cotton Leafworm Spodoptera littoralis (Lepidoptera: Noctuidae)
}

\author{
Ghoneim $\mathrm{K}^{1 *}$, Hamadah $\mathrm{Kh}^{1}$, Selim $\mathrm{Sh}^{2}$, Waheeb $\mathrm{H}^{1}$
}

${ }^{1}$ Department of Zoology and Entomology, Faculty of Science, Al-Azhar University, Cairo, Egypt

${ }^{2}$ Department of Pesticide Chemistry and Technology, Faculty of Desert and Environmental Agriculture, Matrouh University, Matrouh, Egypt

DOI: $10.36347 / \mathrm{sajb} .2021 . v 09 \mathrm{i} 02.004$

| Received: 07.02.2021 | Accepted: 19.02.2021 | Published: 24.02.2021

*Corresponding author: Ghoneim $\mathrm{K}$

Abstract

Original Research Article

Egyptian cotton leafworm, Spodoptera littoralis, is one of the key pests that cause great damage to cotton plant as well as other field and vegetable crops. Plant products, as a promising alternative to the synthetic insecticides, have now been established worldwide. In this work we studied for the first time the insecticidal activity of the sesquiterpene compound, nerolidol, and its effect on growth, development and metamorphosis of $S$. littoralis. The newly moulted larvae of $5^{\text {th }}$ (penultimate) or $6^{\text {th }}$ (last) instar larvae were fed on castor bean leaves previously treated with seven concentrations of Nerolidol $(400,200,100,50,25,12.5 \& 6.25 \mathrm{ppm})$ for $24 \mathrm{hr}$. The most important results could be summarized as follows. Nerolidol exhibited various degrees of insecticidal activity against larvae, pupae and adults, regardless the instar under treatment. Nerolidol was found more toxic after treatment of last instar larvae $\left(\mathrm{LC}_{50}=42.24 \mathrm{ppm}\right)$ than after treatment of penultimate instar larvae $\left(\mathrm{LC}_{50}=50.01 \mathrm{ppm}\right)$. A remarkable reduction of larval weight gain was recorded, in a dose-dependent course. Similarly, the larval growth was drastically suppressed. The larval and pupal durations were significantly prolonged. Some percentages of the treated $5^{\text {th }}$ instar larvae failed to completely moult into the $6^{\text {th }}$ instar, only at the higher three concentrations. Also, some larvae developed into larval-pupal intermediates. Nerolidol exerted a strong inhibitory action on the pupation rate in a dose-dependent course while the adult emergence was partially blocked, only at the higher concentrations. Nerolidol failed to exert anti-morphogenic action on S. littoralis after treatment of $5^{\text {th }}$ instar larvae, but treatment of $6^{\text {th }}$ instar larvae only with the higher two concentrations resulted in an impaired morphogenesis of some pupae.

Keywords: ecdysis, intermediates, malformation, metamorphosis, mortality, moult, pupation, toxicity.

Copyright ( $(2021$ The Author(s): This is an open-access article distributed under the terms of the Creative Commons Attribution 4.0 International License (CC BY-NC 4.0) which permits unrestricted use, distribution, and reproduction in any medium for non-commercial use provided the original author and source are credited.

\section{INTRODUCTION}

Cotton from the genus Gossypium is one of the economic crops. It is one of the major sources of fiber. Also, cotton plants produce a large amount of seeds [1]. These seeds are rich in protein and have been considered as a valuable source of oil and fodder [2]. In addition, cotton plants typically contain high content of gossypol, a terpenoid, within the glands of seed kernels [3]. The Egyptian cotton leaf worm Spodoptera littoralis (Boisd.) (Lepidoptera: Noctuidae) is the most destructive pest of the cotton plant and several field crops, as well as vegetables and several ornamental crops [4-7]. It is distributed in many European countries [8-10], Asia Minor and the Middle East countries [11-13]. Moreover, this insect pest has a very wide host range of at least 90 plant species of economic importance belonging to 44 families [14-16]. Over the years, the number of attacked plants by $S$. littoralis increased to more than 112 species [16, 17-20].

Although different cultural, mechanical and physical control measures have been applied for the management of $S$. littoralis in Egypt, no satisfactory results can be obtained, most farmers, however, prefer using synthetic pesticides for obtaining fast results [21-24]. Over the past 50 years, the widespread and extensive uses of many environmentally-hazardous insecticides had led to the development of quick resistance of $S$. littoralis to the many of these chemicals [25-28], beside to their hazardous residues in the 
Ghoneim K et al., Sch Acad J Biosci, Feb, 2021; 9(2): 36-57

environment [29, 30]. In some detail, the development of $S$. littoralis resistance to the synthetic pyrethroids, carbamates, organophosphorus and other chemical insecticides has been correlated with the development of cross-resistance in many cases [31]. Also, application of synthetic insecticides is expensive [32]. Beside to these problems, many pesticides are insoluble in water, so large quantities of organic solvents are needed and most of these solvents contaminate the ecosystem [33]. Therefore, searching for new alternative, effective and safer for human health, economic animals, non-target organisms and ecosystem, is prerequisite [34-36]. One of the chief components of Integrated Pest Management is the application of plant extracts, oils and secondary metabolites which are included in the class of 'biopesticides', in addition to certain bacteria, viruses, animals, and certain minerals [37, 38]. In this context, botanicals have been used as effective toxicants, growth regulators, antifeedants and repellents against a wide spectrum of agricultural and public health insects [39-42]. These biopesticides are effective alternative to synthetic insecticides because of their low toxicity to humans and animals, low environmental pollution and other applications. They are generally more eco-friendly alternatives for the insect control [43-45].

The monoterpenes, phenylpropenes and sesquiterpenes have reported to exhibit different biological activities against some economic insect pests as they can act as insecticides [46-48], insect growth regulators [49], antifeedants [50] and repellents [51, 52]. On the other hand, few studies were reported in the current literature on the antifeedant and growth inhibitory effects of these plant products against $S$. littoralis [45, 53-55]. In Egypt, few studies [56-58] revealed the insecticidal activities of different monoterpenes, phenylpropenes and sesquiterpenes against larvae of $S$. littoralis.

\section{Nerolidol}

(3,7,11-trimethyl-1,6,10-dodecatrien-3-ol, Molecular Formula: $\mathrm{C}_{15} \mathrm{H}_{26} \mathrm{O}$ ) is known as one of the most important acyclic sesquiterpenes. Chemically, nerolidol exists in two isomers, a trans form and a cis form [59]. It is synthesized as an intermediate in the production of (3E)-4,8-dimethy-1,3,7-nonatriene, a herbivore-induced volatile that protects plants against herbivore attacks and attracts some predatory insects [59]. The (E)-nerolidol was identified as a potent signal that elicits the plant defenses, such as the tea plant [60]. For more information, see Boncan et al. [61], Wroblewska-Kurdyk et al. [62] and Favaris et al. [63]. Different commercial uses of nerolidol are reported for cosmetics [64] and non-cosmetic products [65]. Also, nerolidol is widely used in the food industry as a flavor enhancer in many food products [59]. In medicine, it is currently under testing as a skin penetration enhancer for the transdermal delivery of therapeutic drugs $[65,66]$.
For more information, see Klopell et al. [67], Nogueira Neto et al. [68] and Javed et al. [69].

With regard to the pest control, Nerolidol isomers act as insect attractants [70], antifeedants [71], larvicidal [72] and ovicidal agent [73, 74]. Wróblewska-Kurdyk et al. [75] evaluated the effect of Nerolidol isomers on the host-plant selection behaviour of the peach potato aphid Myzus persicae. In a recent study, Benelli et al. [76] recorded a high toxicity of (E)-nerolidol against the aphid Metopolophium dirhodum. Depending on the results of da Silva et al. [77], Azamax ${ }^{\circledR}$ was more toxic than the oils of Melaleuca leucadendra and (E)-nerolidol against Tetranychus urticae. However, the oils and (E)-nerolidol were more toxic to Plutella xylostella than Azamax ${ }^{\circledR}$. Recently, also, Hamadah et al. [78] recorded some adverse effects of nerolidol on the adult performance and reproductive potential of $S$. littoralis. The objective of the present study was to assess the insecticidal activity of Nerolidol and its effect on growth, development and metamorphosis of $S$. littoralis.

\section{MATERIALS AND METHODS The insect culture}

A sample of Egyptian cotton leaf worm Spodoptera littoralis (Boisd.) (Lepidoptera: Noctuidae) pupae was kindly obtained from the culture of susceptible strain maintained for several generations in Plant Protection Research Institute, Agricultural Research Center, Doqqi, Giza, Egypt. In the laboratory of Insect Physiology, Faculty of Science, Al-Azhar University, Cairo, a culture was established under laboratory controlled conditions $\left(27 \pm 2^{\circ} \mathrm{C}, 65 \pm 5 \%\right.$ R.H., photoperiod $14 \mathrm{~h} \mathrm{~L}$ and $10 \mathrm{~h} \mathrm{D}$ ). Rearing procedure was carried out according to Ghoneim [79] and improved by Bakr et al. [80]. Egg patches were kept in Petri dishes until hatching. The hatched larvae were transferred into glass containers containing a layer of dry saw dust and tightly covered with muslin cloth secured with rubber bands. For feeding, larvae were provided daily with fresh castor bean leaves Ricinus communis. The developed pupae were collected and placed in clean jars provided with a layer of moistened saw dust. All jars had been kept in suitable cages provided with branches of fresh Tafla plant, Nerium oleander, as oviposition sites. The emerged adults were provided with $10 \%$ honey solution on a cotton wick as a food source. Moths were allowed to mate and lay eggs on branches. The egg patches were collected daily, and transferred into Petri dishes for the next generation.

\section{The tested compound and concentration preparation}

The tested nerolidol 98\% (an acyclic Sesquiterpene) in the present study was purchased from ABCR GmbH, Karlsruhe, Germany. It has the chemical name: (cis + trans) $[3,7$, 11-Trimethyl-1, 6, 10-dodecatrien-3-ol] and Formula: $\mathrm{C}_{15} \mathrm{H}_{26} \mathrm{O}$. Five ml of Tween 60 were added (as emulsifier) to $5 \mathrm{ml}$ of ethyl 
alcohol (95\%). Then, these solvents were mixed thoroughly with $5 \mathrm{ml}$ of nerolidol. For obtaining a stock solution, $90 \mathrm{ml}$ of distilled water was added to the mixture for preparing a concentration of $4.8 \%$ nerolidol, emulsion. The stock solution was diluted with distilled water in volumetric flasks for preparation of a series of concentrations: 400.00, 200.00, 100.00, 50.00, 25.00, $12.50 \& 6.25 \mathrm{ppm}$.

\section{Bioassay of nerolidol}

Bioassay of nerolidol was carried out against the newly moulted $5^{\text {th }}$ (penultimate) larvae and newly moulted $6^{\text {th }}$ (last) larvae. Discs of fresh castor bean leaves were dipped in each concentration for 5 minutes and air dried before introduction to larvae as food for 24 $\mathrm{hr}$ under the aforementioned laboratory conditions. Control larvae received leaf discs after dipping in Tween 60 and alcohol (95\%) solution for 5 minutes. Ten replicates of treated and control larvae (one larva/replicate) were kept separately in glass vials. Then, mortality and biological parameters were recorded daily.

\section{Insecticidal activity}

All mortalities of treated and control (larvae, pupae and adults) were recorded every day and corrected according to Abbott's formula [81] as follows:

$$
\% \text { of corrected mortality }=\frac{\begin{array}{c}
\% \text { of test mortality }-\% \text { of control } \\
\text { mortality }
\end{array}}{100-\% \text { of control mortality }} \times 100
$$

The $\mathrm{LC}_{\mathbf{5 0}}$ was calculated for total mortality by Microsoft ${ }^{\circledR}$ office Excel (2007), according to Finny [82].

\section{Growth, development and metamorphosis}

Larval body weight gain: Each individual larva (treated or control) was carefully weighed every day using a digital balance for recording the weight gain as follows:

Initial body weight (before the beginning of experiment) - final body weight (at the end of experiment).

Growth rate: It was calculated according to Waldauer [83] as follows:

Fresh weight gain during the feeding period/Feeding period $x$ mean fresh body weight of larva

Developmental duration and rate: Dempster's equation [84] was applied for calculating the developmental duration, and Richard's equation [85] was used for calculating the developmental rate.

Pupation rate was expressed in $\%$ of the successfully developed pupae.

Adult emergence: number of successfully emerged adults was expressed in \% according to Jimenez-Peydro et al. [86] as follows:

[No. of completely emerged adults / No. of pupae] $\times 100$

Morphogenesis: The deranged metamorphosis and morphogenesis programs were detected and calculated in larval-pupal or pupal-adult intermediates (\%). Also, pupal deformation was calculated in $\%$. Features of impaired programs were recorded in photos.

\section{STATISTICAL ANALYSIS OF DATA}

Data obtained were analyzed by the Student's $t$-distribution, and refined by Bessel correction [87] for the test significance of difference between means using GraphPad InStat ${ }^{\circledR}$ v. 3.01 [88].

\section{RESULTS}

\section{Insecticidal activity of nerolidol against $\boldsymbol{S}$. littoralis}

After treatment of newly moulted penultimate

$\left(5^{\text {th }}\right)$ instar larvae of $S$. littoralis with seven concentration levels of nerolidol, data of the insecticidal activity were summarized in Table (1). According to these data, nerolidol exhibited acute toxicity on the $5^{\text {th }}$ instar larvae only at the higher two concentration levels (50 \& 20\% larval mortality, at $400 \& 200$ ppm, respectively, vs. $0 \%$ mortality of control congeners). Stronger toxicity was observed on the successfully moulted last instar larvae, since mortality \% increased parallel to the concentration level, with an exception of the lowest concentration level (50, 50, 40, 40, $30 \& 20 \%$ larval mortality, at 400, 200, $100,50,25 \& 12.5 \mathrm{ppm}$, respectively, vs. $0 \%$ mortality of control larvae). A weak toxic effect was exhibited by nerolidol on the developed pupae and emerged adult moths (see Table 1). $\mathrm{LC}_{50}$ value was calculated in 50.01 ppm.

After treatment of newly moulted last $\left(6^{\text {th }}\right)$ instar larvae of $S$. littoralis with seven concentration levels of nerolidol, data of the insecticidal activity against all developmental stages were arranged in Table (2). In the light of these data, nerolidol exhibited a considerably toxic effect on larvae, in a dose-dependent course, with an exception of the lowest concentration level $(80,70,60,50,30 \& 20 \%$ larval mortality, at 400, $200,100,50,25 \& 12.5 \mathrm{ppm}$, respectively, in comparison with $0 \%$ mortality of control larvae). Nerolidol displayed a pupicidal effect only after larval treatment with the higher two concentration levels. In 
respect of the successfully emerged adult moths, only the higher three concentration levels caused various degrees of toxicity against adults $(50,50 \& 20 \%$ adult mortality, at $200,100 \& 50 \mathrm{ppm}$, respectively, vs. $0 \%$ mortality of control adults). $\mathrm{LC}_{50}$ value was determined in 42.24 ppm.

In the light of data of both tables $1 \& 2$, nerolidol was found more toxic after treatment of $6^{\text {th }}$ instar larvae than its toxicity after treatment of $5^{\text {th }}$ instar larvae. In other words, the last instar was more sensitive to Nerolidol toxicity than penultimate instar.

\section{Effect of nerolidol on growth, development, metamorphosis and morphogenesis of $\boldsymbol{S}$. littoralis}

After treatment of $5^{\text {th }}$ instar larvae with seven concentration levels of nerolidol, data of weight gain, growth, development, metamorphosis and morphogenesis were assorted in Table (3). Data of similar criteria were arranged in Table (4), after treatment of $6^{\text {th }}$ instar larvae with nerolidol.

\section{Effect on the weight gain and growth}

Data of Table (3) revealed a remarkable reduction of larval weight gain (wtg), after treatment of $5^{\text {th }}$ instar larvae with nerolidol, in a dose-dependent course $\quad(48.19 \pm 2.11, \quad 56.33 \pm 1.74, \quad 62.14 \pm 1.09$, $65.64 \pm 0.98,73.25 \pm 1.76,81.72 \pm 1.09 \& 84.15 \pm 2.05 \mathrm{mg}$, at $400,200,100,50,25,12.5,6.25 \mathrm{ppm}$, respectively, $v s$. $86.19 \pm 2.71 \mathrm{mg}$ of control larvae). Similarly, nerolidol suppressed the larval growth rate (GR), in a dose-dependent course $\quad(3.00 \pm 0.01, \quad 4.34 \pm 0.56$, $5.43 \pm 0.56, \quad 6.19 \pm 0.78, \quad 8.95 \pm 0.16, \quad 9.65 \pm 0.26 \quad \&$ $10.36 \pm 0.42$, at $400,200,100,50,25,12.5,6.25 \mathrm{ppm}$, respectively, vs. $12.49 \pm 0.53$ of control larvae). In addition, wtg of the successfully moulted $6^{\text {th }}$ instar larvae had been subjected to a strong reducing action of nerolidol. Also, these larvae appeared with pronouncedly regressed GR (for detail, see 3).

After treatment of $6^{\text {th }}$ instar larvae with nerolidol, data of Table (4) revealed a drastic reduction of the larval wtg $(142.13 \pm 2.77,162.68 \pm 3.19$, $188.24 \pm 4.08,193.51 \pm 1.19,211.18 \pm 3.67,226.20 \pm 4.15 \&$ $233.14 \pm 0.28 \mathrm{mg}$, at $400,200,100,50,25,12.5,6.25$ ppm, respectively, vs. $234.28 \pm 2.01 \mathrm{mg}$ of control larvae) and considerably regressed GR.

\section{Effect on the developmental durations and rate}

Data of Table (3) revealed a remarkably prolonged larval duration after treatment of $5^{\text {th }}$ instar larvae with only higher three concentration levels of Nerolidol $\quad(48.19 \pm 2.11, \quad 56.33 \pm 1.74, \quad 62.14 \pm 1.09$, $65.64 \pm 0.98,73.25 \pm 1.76,81.72 \pm 1.09 \& 84.15 \pm 2.05$ days of treated larvae, at 400, 200, 100, 50, 25, $12.5 \& 6.25$ ppm, respectively, vs. $2.31 \pm 0.48$ days of control larvae). Also, the successfully moulted $6^{\text {th }}$ instar larvae passed a general prolonged period, but significantly prolonged period only after treatment with the higher two concentration levels of nerolidol $(9.00 \pm 0.33$ \& $8.67 \pm 0.48$ days of treated larvae, at $400 \& 200 \mathrm{ppm}$, respectively, vs. $7.81 \pm 0.67$ days of control larvae). In addition, the successfully developed pupae after treatment of $5^{\text {th }}$ instar larvae with all nerolidol concentration levels, except the lowest one, survived significantly prolonged period $(8.19 \pm 0.36,7.87 \pm 0.16$, $7.74 \pm 0.25,7.49 \pm 0.53 \& 7.28 \pm 0.67$ days of treated pupae, at 200,100,50, $25 \& 12.5 \mathrm{ppm}$, respectively, vs. $6.87 \pm 0.33$ days of control pupae).

A similar prolongation of pupal duration was determined after treatment of $6^{\text {th }}$ instar larvae with nerolidol. This prolonged duration was found in a dose-dependent course $\quad(8.52 \pm 0.47, \quad 8.28 \pm 0.24$, $7.96 \pm 0.10,7.78 \pm 0.25,7.53 \pm 0.42 \& 7.23 \pm 0.34$ days of treated pupae, at $200,100,50,25,12.5 \& 6.25 \mathrm{ppm}$, respectively, vs. $6.87 \pm 0.33$ days of control pupae, see Table 4).

With regard to the developmental rate (DR), data of Table (3) displayed a strong suppressing action of nerolidol on DR of treated $5^{\text {th }}$ instar larvae, proportional to the increasing concentration level. A similar regression of DR was recorded for $6^{\text {th }}$ instar larvae after treatment with nerolidol (for detail, see Table 4).

\section{Effect on the developmental program}

Data of Table (3) included a criterion of the disrupted developmental program, failure of ecdysis. For some detail, some percentages of the treated $5^{\text {th }}$ instar larvae failed to completely moult into the $6^{\text {th }}$ instar, only after treatment with the higher three concentration levels of nerolidol (20, $20 \& 10 \%$ failed larvae to moult, at 400, $200 \& 100 \mathrm{ppm}$, respectively, compared to $0 \%$ failure of control larvae). As shown in Fig. (1), these $6^{\text {th }}$ instar larvae appeared with rudimentary $5^{\text {th }}$ instar exuvia and abdominal constrictions.

Another feature of disrupted developmental program is the production of larval-pupal intermediates. Depending on data of Table (3), treatment of $5^{\text {th }}$ instar larvae with Nerolidol were induced to produce different intermediate creatures. With exception of the lower two concentration levels, these intermediates were produced in increasing percentage with the increasing concentration level $(30,30,20,10 \& 10 \%$ of larval-pupal intermediates, at 400, 200, 100, 50 \& 25 ppm, respectively).

In addition, a similar feature of disrupted developmental program was recorded after treatment of $6^{\text {th }}$ instar larvae. The larval-pupal intermediates were increasingly produced as the concentration level was increased, with exception of the lowest concentration level of nerolidol $(70,50,40,40,20 \& 10 \%$ of intermediates, at 400, 200, 100, 50, 25 \& 12.5ppm, respectively, see Table 4). Irrespective of the larval instar under treatment, the important features of these 
Ghoneim K et al., Sch Acad J Biosci, Feb, 2021; 9(2): 36-57

intermediates had been observed with pupal abdomen and larval head and thorax (see Fig. 2).

\section{Effect on the metamorphosis}

\section{Pupation}

Depending on data assorted in Table (3), nerolidol exerted a strong inhibitory action on pupation, since pupation rate was regressed in a dose-dependent course, after treatment of $5^{\text {th }}$ instar larvae, with exception of the lowest concentration level $(30,40,60,70 \& 80 \%$ pupation, at 200,100, 50, $25 \& 12.5 \mathrm{ppm}$, respectively, $v s .100 \%$ pupation of control congeners). A similar inhibitory effect was exhibited by nerolidol on the pupation after treatment of $6^{\text {th }}$ instar larvae. As obviously seen in Table (4), the pupation blocking increased proportional to the increasing nerolidol concentration level $(20,30,40,50,70 \& 80 \%$ pupation, at 400,200 , $100,50,25 \& 12.5 \mathrm{ppm}$, respectively, vs. $100 \%$ pupation of control congeners).

\section{Adult emergence}

It may be important to mention that the adult emergence is a prerequisite process of the insect metamorphosis. On the basis of data assorted in both tables ( $3 \& 4)$, nerolidol had a weak blocking potency on adult emergence after treatment of $5^{\text {th }}$ instar larvae or $6^{\text {th }}$ instar larvae. For some detail, the adult emergence was partially blocked after treatment of $5^{\text {th }}$ instar larvae only with the higher two concentration levels of nerolidol (66.67 \& 83.33\% adult emergence, at $200 \& 100$ ppm, respectively, vs. $100 \%$ emergence of control congeners, Table 3). Also, treatment of $6^{\text {th }}$ instar larvae only with $200 \mathrm{ppm}$ nerolidol resulted in $66.67 \%$ emerged adults while $100 \%$ emerged adults were recorded at other concentrations (Table 4).

\section{Effect on the morphogenesis program}

As seen in Table (3), nerolidol failed to exert anti-morphogenic action on $S$. littoralis after treatment of $5^{\text {th }}$ instar larvae. On the other hand, treatment of $6^{\text {th }}$ instar larvae only with the higher two concentration levels of Nerolidol impaired the morphogenesis of some pupae $(100.00 \& 33.33 \%$ deformed pupae, at $400 \& 200$ ppm, respectively, vs. $0 \%$ deformation of control pupae, see Table 4). As observed in Fig. (3), the malformed pupae developed with bent abdomens, hump-backs or with last larval exuvia attached to head and mouth parts.

Table-1: Insecticidal activity $(\%)$ of Nerolidol against $S$. littoralis after treatment of newly moulted penultimate $\left(5^{\text {th }}\right)$ instar larvae

\begin{tabular}{|c|c|c|c|c|c|c|c|}
\hline \multirow[b]{2}{*}{$\begin{array}{l}\text { Conc. } \\
\text { (ppm) }\end{array}$} & \multicolumn{2}{|c|}{ Larval mortalities } & \multirow[b]{2}{*}{$\begin{array}{c}\text { Pupal } \\
\text { mortality }\end{array}$} & \multirow[b]{2}{*}{$\begin{array}{c}\text { Adult } \\
\text { mortality }\end{array}$} & \multirow[b]{2}{*}{$\begin{array}{c}\text { Total } \\
\text { mortality }\end{array}$} & \multirow[b]{2}{*}{$\begin{array}{l}\text { Corrected } \\
\text { mortality }\end{array}$} & \multirow[b]{2}{*}{$\begin{array}{c}\mathbf{L C}_{50} \\
(\mathbf{p p m})\end{array}$} \\
\hline & $\begin{array}{c}5^{\text {th }} \\
\text { instar } \\
\end{array}$ & $\begin{array}{c}6^{\text {th }} \\
\text { instar }\end{array}$ & & & & & \\
\hline 400.00 & 50.00 & 100.00 & --- & --- & 100.00 & 100.00 & \multirow{8}{*}{50.01} \\
\hline 200.00 & 20.00 & 62.50 & 33.33 & 0.00 & 80.00 & 80.00 & \\
\hline 100.00 & 0.00 & 40.00 & 0.00 & 16.67 & 70.00 & 70.00 & \\
\hline 50.00 & 0.00 & 40.00 & 16.67 & 0.00 & 50.00 & 50.00 & \\
\hline 25.00 & 0.00 & 30.00 & 0.00 & 0.00 & 30.00 & 30.00 & \\
\hline 12.50 & 0.00 & 20.00 & 0.00 & 0.00 & 20.00 & 20.00 & \\
\hline 6.25 & 0.00 & 0.00 & 0.00 & 0.00 & 0.00 & 0.00 & \\
\hline Control & 0.00 & 0.00 & 0.00 & 0.00 & 0.00 & -- & \\
\hline
\end{tabular}

Table-2: Insecticidal activity (\%) of Nerolidol against $S$. littoralis after treatment of newly moulted last instar larvae

\begin{tabular}{|c|c|c|c|c|c|c|}
\hline $\begin{array}{c}\text { Conc. } \\
\text { (ppm) }\end{array}$ & $\begin{array}{c}\text { Larval } \\
\text { mortality }\end{array}$ & $\begin{array}{c}\text { Pupal } \\
\text { mortality }\end{array}$ & $\begin{array}{c}\text { Adult } \\
\text { mortality }\end{array}$ & $\begin{array}{c}\text { Total } \\
\text { mortality }\end{array}$ & $\begin{array}{c}\text { Corrected } \\
\text { mortality }\end{array}$ & LC $_{\mathbf{5 0}}(\mathbf{p p m})$ \\
\hline 400.00 & 80.00 & 100.00 & --- & 100.00 & 100.00 & \\
\hline 200.00 & 70.00 & 33.33 & 50.00 & 90.00 & 90.00 \\
\hline 100.00 & 60.00 & 0.00 & 50.00 & 80.00 & 80.00 \\
\hline 50.00 & 50.00 & 0.00 & 20.00 & 60.00 & 60.00 \\
\hline 25.00 & 30.00 & 0.00 & 0.00 & 30.00 & 30.00 & 42.24 \\
\hline 12.50 & 20.00 & 0.00 & 0.00 & 20.00 & 20.00 \\
\hline 6.25 & 0.00 & 0.00 & 0.00 & 0.00 & 0.00 \\
\hline Control & 0.00 & 0.00 & 0.00 & 0.00 & -- \\
\hline
\end{tabular}

Conc. ---: see footnote of Table (1). 
Ghoneim K et al., Sch Acad J Biosci, Feb, 2021; 9(2): 36-57

Table-3: Growth and development of $S$. littoralis after treatment of the newly moulted $5^{\text {th }}$ instar larvae with

\begin{tabular}{|c|c|c|c|c|c|c|c|c|c|c|c|c|c|}
\hline \multirow[b]{4}{*}{ 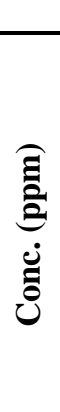 } & \multirow{2}{*}{\multicolumn{9}{|c|}{ Larval instar }} & \multirow{2}{*}{\multicolumn{3}{|c|}{ Pupal stage }} & \multirow[b]{4}{*}{ 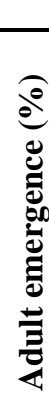 } \\
\hline & & & & & & & & & & & & & \\
\hline & \multicolumn{4}{|c|}{$5^{\text {th }}$} & \multicolumn{5}{|c|}{$6^{\text {th }}$} & \multirow[b]{2}{*}{ 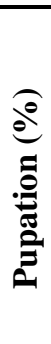 } & \multirow[b]{2}{*}{ 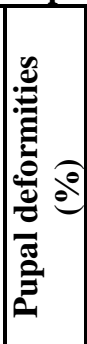 } & \multirow[b]{2}{*}{ 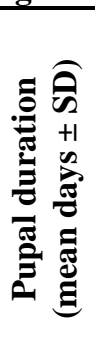 } & \\
\hline & 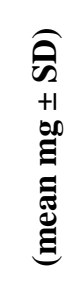 & 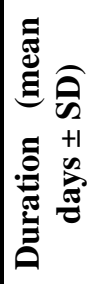 & 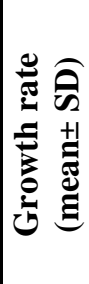 & 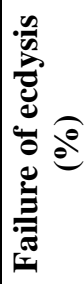 & 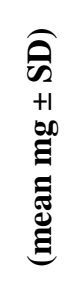 & 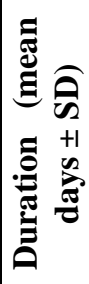 & 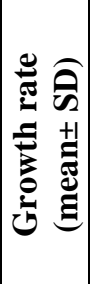 & 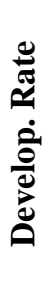 & 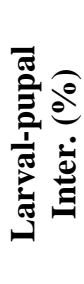 & & & & \\
\hline $\begin{array}{l}8 \\
\dot{8} \\
\dot{q}\end{array}$ & $\begin{array}{l}0 \\
= \\
\dot{+1} \\
\stackrel{+1}{a} \\
\stackrel{\infty}{+}\end{array}$ & 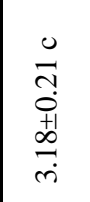 & $\begin{array}{l}0 \\
0 \\
0 \\
0 \\
+1 \\
0 \\
\dot{0}\end{array}$ & $\begin{array}{l}\stackrel{8}{0} \\
\stackrel{\mathrm{d}}{ }\end{array}$ & $\begin{array}{l}\overrightarrow{0} \\
\pm \\
0 \\
+1 \\
\stackrel{+1}{0} \\
\dot{0}\end{array}$ & $\begin{array}{l}0 \\
m \\
0 \\
0 \\
0 \\
0 \\
0\end{array}$ & 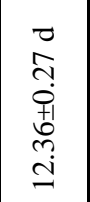 & 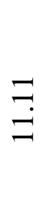 & $\begin{array}{l}\text { \& } \\
\text { @) }\end{array}$ & 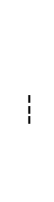 & 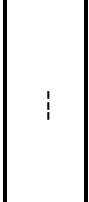 & $i$ & 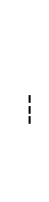 \\
\hline $\begin{array}{l}8 \\
\stackrel{8}{0} \\
\stackrel{\leftrightarrow}{0}\end{array}$ & 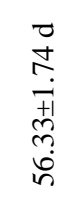 & $\begin{array}{l}0 \\
0 \\
n \\
0 \\
+1 \\
0 \\
0 \\
r\end{array}$ & $\begin{array}{l}\overrightarrow{0} \\
0 \\
\vdots \\
0 \\
+1 \\
+ \\
+ \\
+\end{array}$ & $\begin{array}{l}\stackrel{8}{8} \\
\stackrel{\text { }}{ }\end{array}$ & 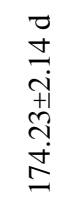 & $\begin{array}{l}0 \\
\infty \\
+ \\
0 \\
+1 \\
60 \\
\infty \\
\infty\end{array}$ & $\begin{array}{l}0 \\
0 \\
0 \\
+1 \\
0 \\
n \\
n \\
n\end{array}$ & $\stackrel{n}{=}$ & 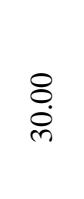 & $\begin{array}{l}8 \\
\stackrel{8}{\circ} \\
\text { ळे }\end{array}$ & $\stackrel{8}{8}$ & $\begin{array}{l}0 \\
0 \\
0 \\
0 \\
+1 \\
0 \\
\infty \\
\infty\end{array}$ & $\begin{array}{l}\hat{\sigma} \\
\dot{\theta}\end{array}$ \\
\hline $\begin{array}{l}8 \\
\stackrel{8}{8} \\
\stackrel{0}{0}\end{array}$ & 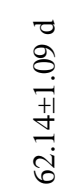 & 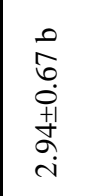 & \begin{tabular}{l}
0 \\
0 \\
$n$ \\
0 \\
+1 \\
\multirow{1}{*}{} \\
$\dot{n}$
\end{tabular} & $\begin{array}{l}8 \\
\stackrel{0}{0}\end{array}$ & $\begin{array}{l}\overrightarrow{0} \\
0 \\
0 \\
i \\
+1 \\
\infty \\
\infty \\
\vdots \\
\vdots \\
0\end{array}$ & $\begin{array}{l}\sigma \\
\hat{0} \\
\dot{0} \\
+1 \\
m \\
\infty \\
\infty\end{array}$ & 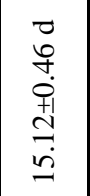 & $\begin{array}{l}\stackrel{8}{ } \\
\text { i }\end{array}$ & $\begin{array}{l}\stackrel{8}{8} \\
\stackrel{\leftrightarrow}{ }\end{array}$ & $\begin{array}{l}\stackrel{8}{8} \\
\stackrel{+}{+}\end{array}$ & $\stackrel{8}{8}$ & $\begin{array}{l}\overrightarrow{0} \\
0 \\
\vdots \\
+1 \\
+1 \\
\infty \\
r\end{array}$ & $\begin{array}{l}m \\
\ddot{\infty}\end{array}$ \\
\hline $\begin{array}{l}8 \\
\stackrel{8}{0} \\
\stackrel{n}{0}\end{array}$ & $\begin{array}{l}0 \\
\infty \\
0 \\
0 \\
+1 \\
+0 \\
\dot{1} \\
\dot{b}\end{array}$ & 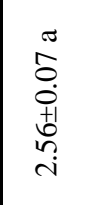 & $\begin{array}{l}0 \\
\infty \\
0 \\
0 \\
+1 \\
0 \\
0\end{array}$ & $\stackrel{8}{8}$ & $\begin{array}{l}0 \\
0 \\
0 \\
m \\
+1 \\
+ \\
+ \\
\dot{d} \\
\dot{d}\end{array}$ & 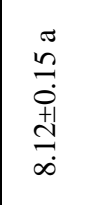 & 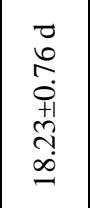 & $\begin{array}{c}\stackrel{\sim}{\sim} \\
\stackrel{\sim}{\sim}\end{array}$ & $\begin{array}{l}8 \\
\stackrel{0}{0}\end{array}$ & $\begin{array}{l}8 \\
\stackrel{8}{8}\end{array}$ & $\stackrel{8}{8}$ & 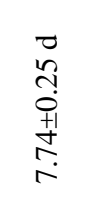 & $\begin{array}{l}8 \\
\stackrel{8}{8} \\
\stackrel{0}{0}\end{array}$ \\
\hline $\begin{array}{l}8 \\
\stackrel{8}{1} \\
\end{array}$ & 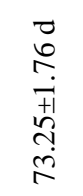 & 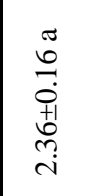 & $\begin{array}{l}0 \\
0 \\
\dot{0} \\
+1 \\
\vdots \\
\infty \\
\infty\end{array}$ & $\stackrel{8}{8}$ & 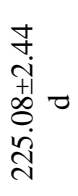 & 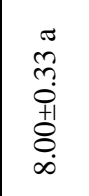 & 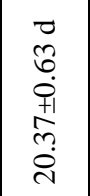 & 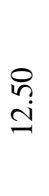 & $\begin{array}{l}8 \\
0\end{array}$ & $\underset{8}{8}$ & $\stackrel{8}{8}$ & $\begin{array}{l}0 \\
n \\
n \\
o \\
+1 \\
o+j \\
r\end{array}$ & $\begin{array}{l}8 \\
\stackrel{8}{8} \\
8\end{array}$ \\
\hline 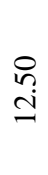 & 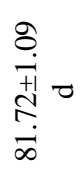 & 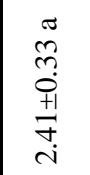 & $\begin{array}{l}0 \\
0 \\
1 \\
0 \\
+1 \\
0 \\
0 \\
0 \\
0\end{array}$ & $\stackrel{8}{8}$ & 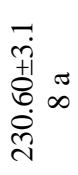 & $\begin{array}{l}\tilde{\sigma} \\
0 \\
+ \\
0 \\
+1 \\
\vdots \\
\\
\end{array}$ & 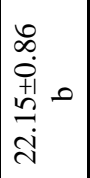 & 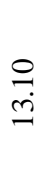 & $\stackrel{8}{8}$ & $\begin{array}{l}8 \\
\stackrel{\infty}{\infty}\end{array}$ & $\stackrel{8}{8}$ & 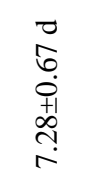 & $\begin{array}{l}8 \\
\dot{8} \\
8\end{array}$ \\
\hline ๙ֶ) & $\begin{array}{l}\pi \\
\check{0} \\
i \\
+1 \\
n \\
\dot{+} \\
\infty\end{array}$ & 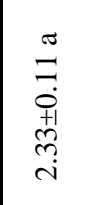 & 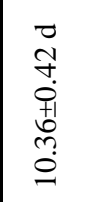 & $\stackrel{8}{0}$ & 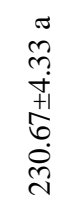 & $\begin{array}{l}\approx \\
0 \\
\infty \\
0 \\
+1 \\
0 \\
\infty \\
\infty \\
r\end{array}$ & 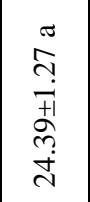 & $\begin{array}{l}\text { ஸे } \\
\stackrel{2}{ }\end{array}$ & $\stackrel{8}{8}$ & $\begin{array}{l}8 \\
\stackrel{8}{8}\end{array}$ & $\stackrel{8}{8}$ & $\begin{array}{l}\pi \\
m \\
\ddot{0} \\
+1 \\
0 \\
0\end{array}$ & $\begin{array}{l}8 \\
\stackrel{8}{8} \\
\stackrel{0}{0}\end{array}$ \\
\hline ن롤 & 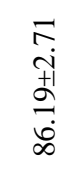 & 京 & 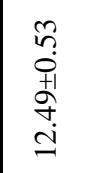 & $\stackrel{8}{8}$ & $\begin{array}{l}n \\
n \\
i \\
+1 \\
0 \\
0 \\
0 \\
\infty \\
0\end{array}$ & $\begin{array}{l}\hat{\sigma} \\
0 \\
+1 \\
\infty \\
\infty\end{array}$ & 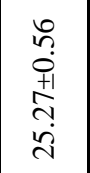 & 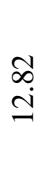 & $\stackrel{8}{8}$ & $\begin{array}{l}8 \\
\stackrel{8}{8} \\
\stackrel{0}{0}\end{array}$ & $\stackrel{8}{8}$ & 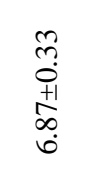 & $\begin{array}{l}8 \\
\stackrel{8}{8} \\
\stackrel{0}{0}\end{array}$ \\
\hline
\end{tabular}

Conc., ---: See footnote of Table (1). Develop. Developmental. Inter.: Intermediate. Mean \pm SD followed with letter: a: insignificant (P $>0.05)$, b: significant $(\mathrm{P}<0.05)$, c: highly significant $(\mathrm{P}<0.01)$, d: extremely significant $(\mathrm{P}<0.001)$ 
Ghoneim K et al., Sch Acad J Biosci, Feb, 2021; 9(2): 36-57

Table-4: Growth and development of $S$. littoralis after treatment of the newly moulted $6^{\text {th }}$ instar larvae with Nerolidol

\begin{tabular}{|c|c|c|c|c|c|c|c|c|c|}
\hline \multirow[b]{2}{*}{$\begin{array}{l}\text { Conc. } \\
\text { (ppm) }\end{array}$} & \multicolumn{5}{|c|}{ Larval instar } & \multicolumn{3}{|c|}{ Pupal stage } & \multirow[b]{2}{*}{$\begin{array}{c}\text { Adult } \\
\text { emergence } \\
(\%)\end{array}$} \\
\hline & $\begin{array}{c}\text { Weight } \\
\text { gain } \\
\text { (mean mg } \pm \\
\text { SD) }\end{array}$ & $\begin{array}{c}\text { Duration } \\
\text { (mean } \\
\text { days } \pm \\
\text { SD) }\end{array}$ & $\begin{array}{l}\text { Growth } \\
\text { rate } \\
\text { (mean } \pm \\
\text { SD) }\end{array}$ & $\begin{array}{l}\text { Develop. } \\
\text { Rate }\end{array}$ & $\begin{array}{c}\text { Larval- } \\
\text { pupal } \\
\text { Inter. } \\
(\%)\end{array}$ & $\begin{array}{c}\text { Pupation } \\
(\%)\end{array}$ & $\begin{array}{c}\text { Pupal } \\
\text { deformities } \\
(\%)\end{array}$ & $\begin{array}{c}\text { Pupal } \\
\text { duration } \\
\text { (mean } \\
\text { days } \pm \\
\text { SD) }\end{array}$ & \\
\hline 400.00 & $\begin{array}{c}142.13 \pm 2.77 \\
\mathrm{~d}\end{array}$ & $\begin{array}{c}9.17 \pm 0.08 \\
\mathrm{~d}\end{array}$ & $\begin{array}{c}9.26 \pm 0.75 \\
\mathrm{~d}\end{array}$ & 10.91 & 70.00 & 20.00 & 100.00 & --- & --- \\
\hline 200.00 & $\begin{array}{c}162.68 \pm 3.19 \\
\mathrm{~d}\end{array}$ & $\begin{array}{c}9.05 \pm 0.33 \\
\mathrm{~d}\end{array}$ & $\begin{array}{c}11.58 \pm 0.36 \\
\mathrm{~d}\end{array}$ & 11.05 & 50.00 & 30.00 & 33.33 & $\begin{array}{c}8.52 \pm 0.47 \\
\mathrm{~d}\end{array}$ & 66.67 \\
\hline 100.00 & $\begin{array}{c}188.24 \pm 4.08 \\
\mathrm{~d}\end{array}$ & $\begin{array}{c}8.56 \pm 0.41 \\
\mathrm{~d}\end{array}$ & $\begin{array}{c}14.44 \pm 0.57 \\
\mathrm{~d}\end{array}$ & 11.68 & 40.00 & 40.00 & 0.00 & $\begin{array}{c}8.28 \pm 0.24 \\
d\end{array}$ & 100.00 \\
\hline 50.00 & $\begin{array}{c}193.51 \pm 1.19 \\
\mathrm{~d}\end{array}$ & $\begin{array}{c}8.33 \pm 0.11 \\
\mathrm{c}\end{array}$ & $\begin{array}{c}16.67 \pm 0.33 \\
\mathrm{~d}\end{array}$ & 12.00 & 40.00 & 50.00 & 0.00 & $\begin{array}{c}7.96 \pm 0.10 \\
\mathrm{~d}\end{array}$ & 100.00 \\
\hline 25.00 & $\begin{array}{c}211.18 \pm 3.67 \\
\mathrm{~d}\end{array}$ & $\begin{array}{c}7.74 \pm 0.21 \\
b\end{array}$ & $\begin{array}{c}19.56 \pm 0.39 \\
d\end{array}$ & 12.92 & 20.00 & 70.00 & 0.00 & $\begin{array}{c}7.78 \pm 0.25 \\
\mathrm{~d}\end{array}$ & 100.00 \\
\hline 12.50 & $\begin{array}{c}226.20 \pm 4.15 \\
\mathrm{~d}\end{array}$ & $\begin{array}{c}7.67 \pm 0.52 \\
\mathrm{a}\end{array}$ & $\begin{array}{c}22.18 \pm 0.81 \\
b\end{array}$ & 13.04 & 10.00 & 80.00 & 0.00 & $\begin{array}{c}7.53 \pm 0.42 \\
\mathrm{~d}\end{array}$ & 100.00 \\
\hline 6.25 & $\begin{array}{c}233.14 \pm 0.28 \\
\mathrm{a}\end{array}$ & $\begin{array}{c}7.56 \pm 0.33 \\
\mathrm{a}\end{array}$ & $\begin{array}{c}23.46 \pm 0.37 \\
b\end{array}$ & 13.23 & 0.00 & 100.00 & 0.00 & $\begin{array}{c}7.23 \pm 0.34 \\
\mathrm{c}\end{array}$ & 100.00 \\
\hline Control & $234.28 \pm 2.01$ & $7.20 \pm 0.63$ & $25.27 \pm 0.56$ & 13.89 & 0.00 & 100.00 & 0.00 & $6.87 \pm 0.33$ & 100.00 \\
\hline
\end{tabular}

Conc.: see footnote of Table (1). Develop.. Inter., a, b, c, d: see footnote of Table (3).

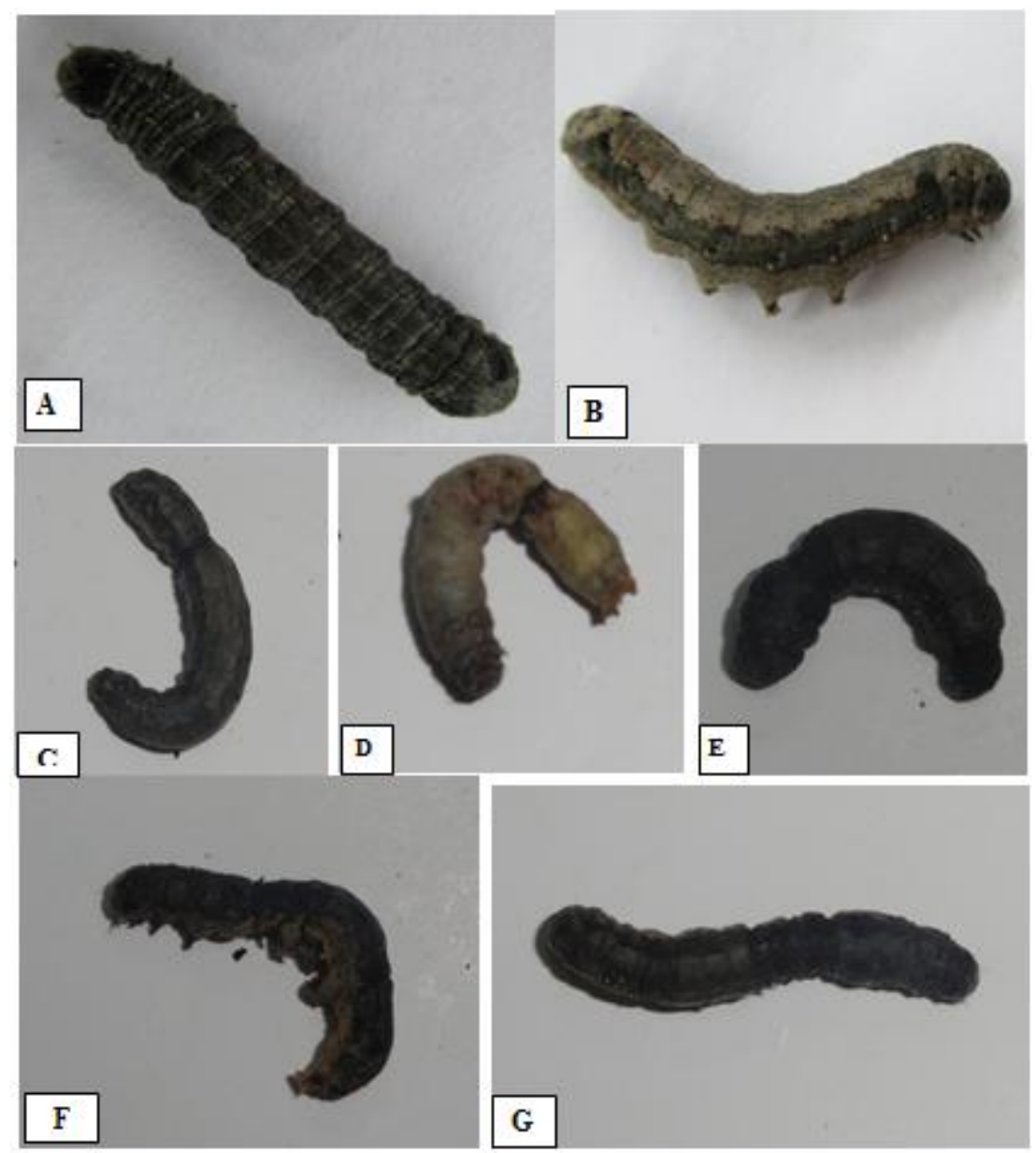

Fig-1: Failure of ecdysis of $S$. littoralis $5^{\text {th }}$ instar larvae after treatment with higher concentrations of Nerolidol. (A): Normal $5^{\text {th }}$ instar larva. (B) Normal $6^{\text {th }}$ instar larva. (C, D, E, F \& G): Various symptoms of incompletely moulted $6^{\text {th }}$ instar larvae with old $5^{\text {th }}$ instar cuticles and abdominal constrictions. 


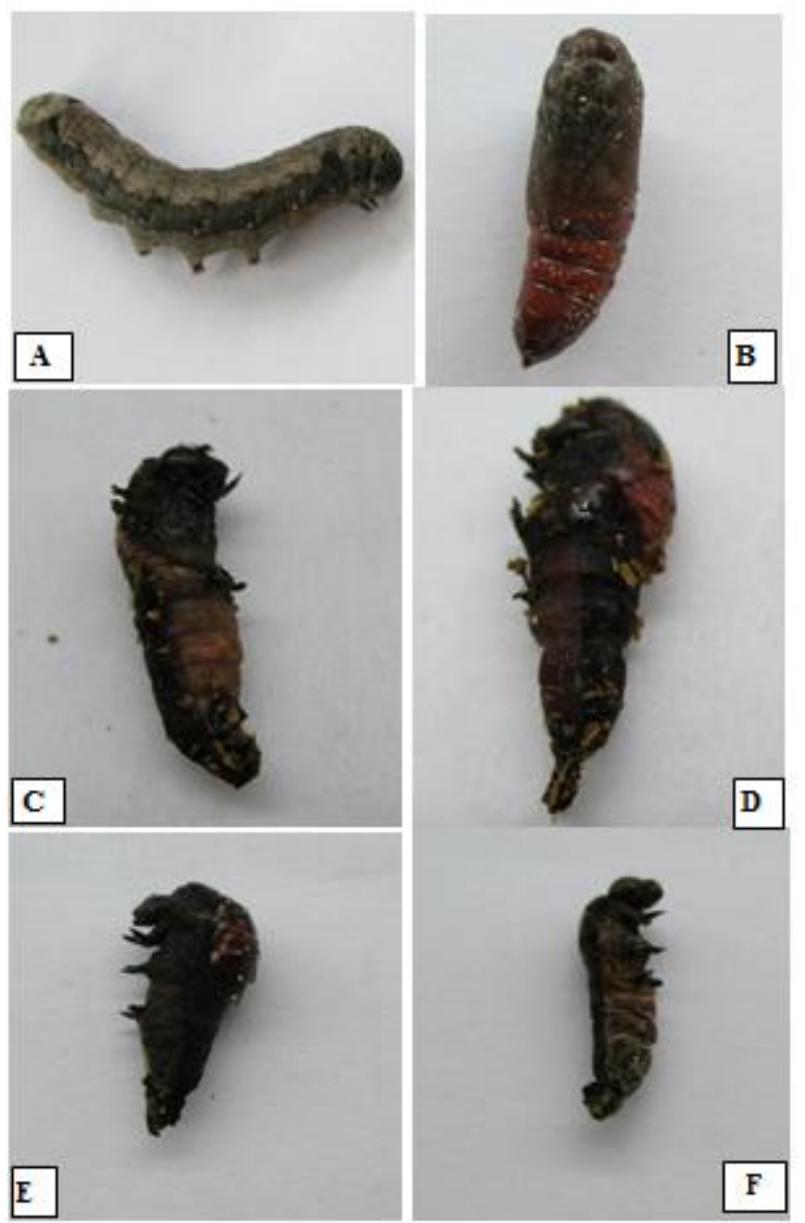

Fig-2: Larval-pupal intermediates of $S$. littoralis as features of disturbed metamorphosis program by Nerolidol, regardless the concentration or larval instar under treatment. (A) Normal last instar larva. (B) Normal pupa. (C, D, E \& F): Various larval-pupal intermediates (pupal abdomen with larval thorax and head).

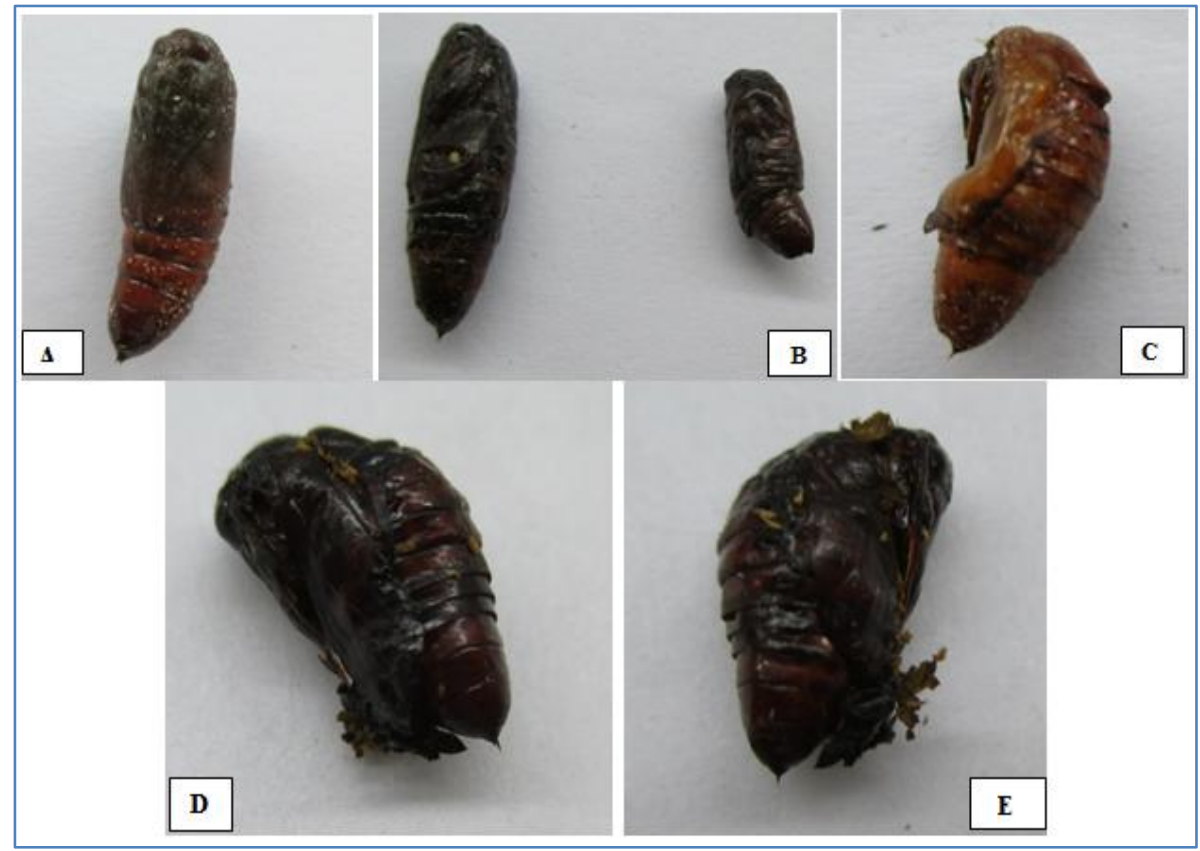

Fig-3: Pupal deformations of $S$. littoralis produced by Nerolidol after treatment of last instar larvae with higher concentrations. (A) Normal pupa. (B) Normal pupa (at left) and dwarf pupa with bent abdomen (at right). (C) Hump- back pupa. (D \& E) Hump-back pupa with last larval cuticle attached to head and mouth parts 
Ghoneim K et al., Sch Acad J Biosci, Feb, 2021; 9(2): 36-57

\section{DISCUSION}

\section{Biopesticidal potential of nerolidol against $S$. littoralis}

Different monoterpenes, phenylpropenes and sesquiterpenes had been reported to exhibit insecticidal activities against Spodoptera littoralis [20, 45, 56, 58, $89,90]$. For examples, 5,6-dihydroxy-3,4-7 trimethoxy flavones (isolated from Artemisia maritima) was found to be toxic against $2^{\text {nd }}$ and $4^{\text {th }}$ instar larvae of $S$. littoralis [91]. The trans-ethyl cinnamate, thymol, carvacrol, trans-anethole and piperitone revealed contact toxicities against the $3^{\text {rd }}$ larval instar of $S$. littoralis $[56,89]$. Also, $\gamma$-terpinene and terpinen-4-ol caused contact toxicities against the $4^{\text {th }}$ larval instar of $S$. littoralis $[56,57]$ and $(-)$-carvone and 1,8-cineole showed strong contact toxicities against the $3^{\text {rd }}$ larval instar of S. littoralis [58]. Toxicity of linoleic acid against the larvae of $S$. littoralis was reported by Yousef et al. [92]. Different isolated compounds from the essential oil of Schinus terebinthifolius, such as $\alpha$-pinene, $\alpha$-terpinene, $\beta$-ocimene, limonene, terpinen-4-ol $\alpha$-terpineol, citronellol, thymol and carvacrol had high insecticidal activities against $S$. littoralis [93, 94]. Pavela [89] evaluated the acute toxicity of 32 volatile compounds against $3^{\text {rd }}$ instar larvae of $S$. littoralis and reported that $\alpha$-pinene, $p$-cymene, $\gamma$-terpinene, thymol and carvacrol (applied at $300 \mu \mathrm{g} / \mathrm{larva}$ ) caused $100 \%$ mortality within $24 \mathrm{hr}$. As recorded by Pavela et al. [90], thymol, carvacrol, geranyl acetate, (E)-Nerolidol or phenolic monoterpenes showed significant toxic effects on larvae of S. littoralis. Recently, Abdelgaleil et al. [45] found Cuminaldehyde, $(-)$-carvone and 1,8-cineole as highly active toxicants against the $2^{\text {nd }}$ larval instar of $S$. littoralis. Recently, also, Hamadah, et al. [78] treated the newly moulted larvae of $5^{\text {th }}$ and $6^{\text {th }}$ instar larvae of $S$. littoralis with seven concentrations of nerolidol. This sesquiterpene compound exhibited an adulticidal activity, only at the higher concentrations.

In addition to $S$. littoralis, various plant products had been reported to exhibit toxicities against different insects, such as Pogostone against Spodoptera litura and Spodoptera exigua [95]; Biostop Moustiques ${ }^{\circledR}$ against $4^{\text {th }}$ instar larvae of susceptible and resistant strains of the mosquito Anopheles gambiae [96]; some sesquiterpene lactones and monoterpenoids against $4^{\text {th }}$ instar larvae of the fly Bradysia odoriphaga [97]; carvacrol, (-)- $\alpha$-bisabolol and chamazulene against Diaphorina citri [98]; some Sesquiterpene lactone compounds against Spodoptera frugiperda [99], as well as Azamax ${ }^{\circledR}$ was more toxic than the oils of Melaleuca leucadendra and (E)-nerolidol against Tetranychus urticae. However, the oils and (E)-nerolidol were more toxic to Plutella xylostella than Azamax ${ }^{\circledR}$ [77]. Also, Tang et al. [100] recorded a high toxicity of Concanavalin, a legume lectin, against the potato psyllid Bactericera cockerelli. Cinnamon oil and its components had the highest contact toxicity against Drosophila suzukii, whereas lemongrass oil, its main components, and farnesol were less toxic, and geraniol was the least toxic [101].

Results of the present study were, to a great extent, in agreement with the previously reported results, because treatment of newly moulted penultimate $\left(5^{\text {th }}\right)$ instar larvae with nerolidol resulted in different mortalities of the treated larvae, only at the higher two concentrations. Moreover, the tested compound exhibited stronger toxicity against the successfully moulted last instar larvae while a weak toxic effect was exhibited on the developed pupae and emerged adult moths. In addition, treatment of newly moulted last $\left(6^{\text {th }}\right)$ instar larvae with nerolidol resulted in a considerable larval mortality, in a dose-dependent course. Also, this compound displayed a pupicidal effect only at the higher two concentrations. Nerolidol caused various degrees of toxicity against adults, only at the higher concentrations.

Also, the present results were in corroboration with some reported results of the insecticidal activity of another sesquiterpene compound, Farnesol, against various insects and mites $[102,103]$. For examples, the $(\mathrm{E}, \mathrm{E})-\alpha-$ Farnesene and a mixture of Farnesol isomers caused considerable toxicities against nymphs of the black bean aphid Aphis fabae and the peach potato aphid Myzus persicae [104]; Farnesol (isolated from Stellera chamaejasma) was recorded with remarkably insecticidal activity against the aphids Aphis craccivora and Leucania separata [105]; Awad [106] reported that Farnesol showed a significant dose-dependent increase in mortality of the black cutworm Agrotis ipsilon $4^{\text {th }}$ instar larvae; the high dose of Farnesol reduced the survival of the nymphs of the red cotton stainer bug Dysdercus koenigii to $70 \%$ after $24 \mathrm{~h}$ of exposure and increasing mortality during subsequent days [107]. Recently, Ghoneim et al. [108] recorded serious insecticidal activity of Farnesol against different development stages of $S$. littoralis. For the pure compounds (isolated from Warburgia ugandensis extracts), the larger grain borer Prostephanus truncates was most susceptible to polygodial and warbuganal which caused 64.3 and $61.7 \%$ deaths, respectively [109].

To interpret the insecticidal activity of nerolidol against $S$. littoralis, in the present investigation, some suggestions could be provided. The larval mortality may be attributed to the failure of larvae to moult owing to the inhibition of chitin synthesis $[110,111]$. The larval mortality may be attributed to the inability of moulting larvae to swallow volumes of air for splitting the old cuticle and expand the new one during ecdysis [112]. Also, the larval deaths might be due to the prevented feeding and continuous starvation [113]. The pupal mortality in S. littoralis, in the current study, could be directly or indirectly relate to activities of nerolidol against some vital processes, such as suffocation, bleeding and desiccation owing to imperfect exuvation, 
Ghoneim K et al., Sch Acad J Biosci, Feb, 2021; 9(2): 36-57

failure of vital homeostatic mechanisms, etc. [114]. The adult mortality of $S$. littoralis could be explained by the retention and distribution of nerolidol in the insect body as a result of direct and rapid transport via the haemolymph to other tissues, and/or by lower detoxification capacity of adults against the tested compound [115].

Also, it may be important to explicate the toxicity of nerolidol, in the present work, leading to mortality of larvae, pupae and/or adults of S. littoralis, by its inhibition of Acetylcholinesterase (AchE), one of the most recognized insecticidal mechanisms, since many terpenoid compounds have been reported to inhibit AchE activity in insects resulting in death [116-119]. Moreover, toxicity of the tested Sesquiterpene compound, nerolidol, can be mediated through: i) inhibition of AchE activity which leads ultimately to impaired neurotransmission, ii) depletion of the activity of antioxidant enzymes leading to accumulation of Reactive oxygen species and peroxidation of membrane lipids and iii) Binding to octopamine receptors or GABA-gated chloride channels and iv) inhibition of cytochrome P450-mediated detoxification [120-122]. In addition, nerolidol might induce the apoptosis in $S$. littoralis midgut cells leading to death [100].

With regard to $\mathrm{LC}_{50}$ values of nerolidol against S. littoralis, in the current study, the tested compound was remarkably more toxic after treatment of last $\left(6^{\text {th }}\right)$ instar larvae than treatment of penultimate $\left(5^{\text {th }}\right)$ instar larvae. In other words, the last instar larvae were more susceptible to the insecticidal potency of nerolidol than the penultimate instar larvae. This result based on the $\mathrm{LC}_{50}$ values which were determined in $42.24 \mathrm{ppm}$ and $50.01 \mathrm{ppm}$, respectively. A similar result was reported for the same insect by Ghoneim et al. [108] since Farnesol exhibited stronger insecticidal activity after treatment of $6^{\text {th }}$ instar larvae $\left(\mathrm{LC}_{50}=33.67 \mathrm{ppm}\right)$ than treatment of $5^{\text {th }}$ instar larvae $\left(\mathrm{LC}_{50}=36.56 \mathrm{ppm}\right)$. On the other hand, results of the current study on $S$. littoralis revealed that the $6^{\text {th }}$ instar larvae were more sensitive to nerolidol than $5^{\text {th }}$ instar larvae. The present result disagreed with many reported results on insects, in particular Lepidoptera, since the earlier larval instars had been recorded more sensitive to the toxicity of different plant compounds than the later larval instars. Unfortunately, there is no conceivable interpretation of this finding right now!! However, different $\mathrm{LC}_{50}$ values had been determined for various plant products against several insects. For examples, some naphtooquinone derivatives exhibited different toxicities on $S$. littoralis larvae and isovalerylshikonin was significantly more toxic $\left(\mathrm{LD}_{50}=0.8 \mu \mathrm{g} / \mathrm{cm}^{2}\right)$ than isobutyrylshikonin $\left(\mathrm{LD}_{50}=\right.$ $\left.7.3 \mu \mathrm{g} / \mathrm{cm}^{2}\right)$ [19]. Farnesol exhibited toxicity against $A$. craccivora and $L$. separata, with $\mathrm{LC}_{50}$ values of 20.2 and $15.2 \mathrm{mg} \mathrm{L}^{-1}$, respectively [105]. The plant compounds, 1-desacetylwilforgine, wilforgine, 1-desacetylwilforine and wilforine showed insecticidal activities to the $3^{\text {rd }}$ instar larvae of mosquito Culex pipiens, with $\mathrm{LC}_{50}=$ $25.70,25.40,22.58$ and $14.57 \mu \mathrm{g} / \mathrm{ml}$, respectively and to adults of Musca domestica, with $\mathrm{LC}_{50}=87.29,70.19$, 47.80 and $21.00 \mu \mathrm{gg} / \mathrm{ml}$, respectively [123]. Among eleven terpene ketones, thymoquinone exhibited the highest toxicity against adults of Sitophilus zeamais, with $\mathrm{LC}_{50}=16.5 \mu \mathrm{g} / \mathrm{cm}^{2}$ and $\mathrm{LC}_{50} 13.8 \mu \mathrm{L} / \mathrm{L}$ air $(24 \mathrm{hr}$ after treatment) of contact and fumigant methods, respectively [124]. As reported by AlShebly et al. [125], epi- $\beta$-bisabolol showed high toxicity against the early $3^{\text {rd }}$ instar larvae of the mosquito Anopheles stephensi $\left(\mathrm{LC}_{50}=14.68 \mu \mathrm{g} / \mathrm{ml}\right)$, the mosquito Aedes aegypti $\left(\mathrm{LC}_{50}=15.83 \mu \mathrm{g} / \mathrm{ml}\right)$ and the mosquito Culex quinquefasciatus $\left(\mathrm{LC}_{50}=17.27 \mu \mathrm{g} / \mathrm{ml}\right)$. Baranitharan et al. [126] isolated Methyl 4-piperidineacetate among identified seven compounds in the ethanolic extract of Punica granatum. After treatment of $3^{\text {rd }}$ instar larvae of the mosquito $C$. quinquefasciatus, $\mathrm{LC}_{50}$ was found to be $110.36 \mathrm{ppm}$. In a recent study of Benelli et al. [76], Phytol was the most effective against the aphid Metopolophium dirhodum $\left(\mathrm{LC}_{50}=1.4 \mathrm{~mL} \mathrm{~L}^{-1}\right)$, followed by (E)-nerolidol $\left(\mathrm{LC}_{50}=3.5 \mathrm{~mL} \mathrm{~L}^{-1}\right)$ and spathulenol $\left(\mathrm{LC}_{50}=4.3 \mathrm{~mL} \mathrm{~L}^{-1}\right)$. It may be important to mention that the $\mathrm{LC}_{50}$ values depend on several factors, such as susceptibility of the insect and its treated stage or instar, lethal potency of the tested compounds or products and their concentrations, method and time of treatment or exposure, as well as the experimental conditions [108, 127].

\section{Reduced weight gain and inhibited growth of $S$. littoralis by nerolidol}

Some plant products were reported to reduce the weight of larval, pupal and adult stages of various insects [128]. In the present study on S. littoralis, a remarkable reduction of larval weight gain was recorded after treatment of $5^{\text {th }}$ instar or $6^{\text {th }}$ instar larvae with the sesquiterpene compound, nerolidol, in a dose-dependent course. This result was in agreement with many reported results of reduced larval body weight of $S$. littoralis after treatment with Farnesol [108] and Linoleic acid (= omega-6 fatty acid) [92] or allyl cinnamate $0.05 \%$ [129]. Feeding of $S$. littoralis larvae and the migratory locust Locusta migratoria nymphs on diet treated with Gibberellic acid $\left(\mathrm{GA}_{3}\right)$ resulted in remarkably reduced larval body weight in both insects [130]. In addition, feeding of $A$. ipsilon larvae on a food plant treated with Farnesol, the larval body weight was reduced [131]. The body weight gain of the lesser mealworm Alphitobius diaperinus larvae was reduced after feeding on diet treated with $\beta$-damascone (isolated from Bulgarian rose oil) or its synthetic derivatives $\gamma$ - and $\delta$-halolactones [132].

To explicate the reduction of weight gain of $S$. littoralis larvae after treatment with nerolidol, in the current study, the treated larvae might suffer gut alterations, suggesting that such larvae stopped feeding and consequently lost weight [4]. Another suggestion is 
a post-ingestion toxic effect of nerolidol, causing poor utilization of food by these larvae or inhibiting important vital processes, causing the weight loss [133].

With regard to the growth, different plant products were reported to exhibit inhibitory effects on the growth of insect larvae [128]. In the present study, nerolidol was found a strong growth inhibitor against $S$. littoralis, after treatment of $5^{\text {th }}$ instar or $6^{\text {th }}$ instar larvae. This result was consistent with the reported results of inhibited growth of the same insect after treatment with different plant products. For example, some triterpenes caused growth inhibition of $S$. littoralis larvae, such as limonoids from Khaya senegalensis, Chukrasia tabularis and Swietenia mahogany [5, 134]. Various monoterpenes, phenylpropenes, sesquiterpenes and some terpenoid compounds showed inhibitory effects on the growth of $S$. littoralis [20, 54, 135, 136, 137]. Isobutyrylshikonin and isovalerylshikonin inhibited the growth of S. littoralis larvae [19]. After treatment of $5^{\text {th }}$ or $6^{\text {th }}$ instar larvae of $S$. littoralis with Farnesol, serious reduction of the larval growth rate [108]. Abdelgaleil et al. [45] evaluated the growth inhibitory activities of seven monoterpenes, two phenylpropenes and two sesquiterpenes on $2^{\text {nd }}$ instar larvae of $S$. littoralis. All compounds drastically inhibited the growth of larvae. The cuminaldehyde, 1,8-cineole and eugenol were the most potent growth inhibitors.

Apart from S. littoralis, many studies recorded different inhibitory effects of various plant compounds on the larval growth of some insects. For examples, treatment of the early larvae of $S$. frugiperda with gedunin, photogedunin or Toosendanin resulted in the larval growth inhibition, in a dose-dependent course [138]. The growth inhibition in Bactrocera cucurbitae larvae was documented in dose-dependent course by Kaur and Rup [139] after treatment with Gibberellic acid $\left(\mathrm{GA}_{3}\right)$ or Coumarin, kinetin, $\mathrm{GA}_{3}$ and 3-indoleacetic acid. Feeding of $S$. litura larvae on an artificial diet fortified with Miraculan resulted in suppression of larval growth [140]. Corzo et al. [141] recorded regressed growth rate of $S$. frugiperda larvae by feeding on some sesquiterpenoids. Szołyga et al. [142] showed that $\alpha$ - and $\beta$-thujone inhibited the growth of $A$. diaperinus. Treatment of $S$. frugiperda larvae with Jasmonic acid reduced the larval growth [143]. Treatment of $3^{\text {rd }}$ instar larvae of $S$. litura with Alantolactone and isoalantolactone, and two eudesmane-type sesquiterpene lactones resulted in the inhibition of larval growth [144]. It was also reported that the eudesmane sesquiterpenes inhibited the growth of $S$. frugiperda [145]. Our result was in corroboration with the previously reported results but disagreed with few studies which recorded some inducing effects of certain plant compounds on the larval growth of some insects, such as cucurbitacin-C (an oxygenated triterpene) which had been appeared to promote the growth of S. exigua larvae [146].
The explication of growth inhibition of $S$. littoralis larvae by nerolidol, in the current study, could be provided as follows. The growth inhibition might be a result of the retardation and/or delay in release of certain peptides from neurohaemal organs, causing alteration in the hemolymph ecdysteroid and juvenoid titers [147].

\section{Prolonged developmental durations and retarded developmental rate of $\boldsymbol{S}$. littoralis by nerolidol}

In the present study, a remarkably prolonged larval duration was recorded after treatment of $5^{\text {th }}$ instar or $6^{\text {th }}$ instar larvae with only higher three concentrations of the sesquiterpenoid compound, nerolidol. Also, the developed pupae lived significantly prolonged duration after treatment of $5^{\text {th }}$ instar larvae with nerolidol. The compound exerted a strong suppressing action on the developmental rate, regardless the larval instar under treatment. These results were, to some extent, in agreement with many reported results of prolonged larval and/or pupal duration in different insects after treatment with some plant compounds, such as $S$. littoralis after treatment with 5,6-dihydroxy-3,4-7 trimethoxy flavone [91]; A. ipsilon after feeding on leaves sprayed with Farnesol [131]; S. litura larvae after feeding on an artificial diet fortified with Miraculan [140] and after treatment with higher concentrations of Alantolactone and isoalantolactone [144] or Erucin (4-Methylthiobutyl isothiocyanate) [148]; Schistocerca gregaria after feeding on clover leaves treated with Farnesol [102]; Galleria mellonella larvae after injection of Abscisic acid into the haemocoel [149]; A. diaperinus after feeding on diet treated with $\beta$-damascone or its synthetic derivatives $\gamma$ - and $\delta$-halolactones [41]. Recently, Ghoneim et al. [108] treated the $5^{\text {th }}$ or $6^{\text {th }}$ instar larvae of $S$. littoralis with Farnesol and recorded remarkably prolonged larval and pupal durations, in a dose-dependent course. In contrast, the present results disagreed with some reported results of significantly shortened larval and pupal durations after treatment with some plant compounds, such as $S$. litura and $S$. exigua after treatment with Pogostone [95] and the domestic mosquito C. pipiens after treatment with Saponin [150].

In the present study, prolongation of the larval and pupal durations and retarded development of $S$. littoralis, after larval treatment with nerolidol, could be interpreted by some scenarios. Nerolidol might indirectly interfere with the neuroendocrine organs responsible for the synthesis and release of tropic hormones, like prothoracicotropic hormone [151]. The final step of chitin biosynthesis pathway could be inhibited by nerolidol and the precursor was not converted into chitin for moulting leading to a prolongation of the developmental duration [152]. Also, the prolongation of larval duration might be due to reduced food intake, caused by phagodeterrence of nerolidol [153], or by a deviation of part of the taken food to the detoxification metabolism [154]. With decreased food ingestion and low biomass conversion, 
the insect takes longer to reach the critical weight for ecdysis, leading to the prolongation of larval duration [133]. In addition, nerolidol might exhibit a delaying effect on the pupal transformation into adults [112]. In other words, the prolongation of pupal duration might be due to an elevated titer of juvenile hormone in the haemolymph. Only in the absence of JH in haemolymph, ecdysone could be activated and led to the production of the next stage [155].

\section{Disrupted developmental program of $S$. littoralis by nerolidol \\ Ecdysis failure of larvae}

The failure of larval ecdysis is a criterion of the disrupted developmental program in insects. Depending on the currently available literature, no information was found on this development criterion, as an effect of sesquiterpene compounds or other plant compounds, except a study of Ghoneim et al. [108] who reported that some $5^{\text {th }}$ instar larvae of S. littoralis $(20 \%)$ failed to completely moult into the next instar, after treatment only with the highest concentration level (400 ppm) of Farnesol. A similar result was recorded in the present study, since some percentages of the treated $5^{\text {th }}$ instar larvae failed to completely moult into the $6^{\text {th }}$ instar, only after treatment with the higher three concentrations of nerolidol. These $6^{\text {th }}$ instar larvae were observed with rudimentary $5^{\text {th }}$ instar exuvia and abdominal constrictions.

For the interpretation of this ecdysis failure of treated $S$. littoralis larvae, it may be important to mention that the moulting hormone "ecdysone" plays a key role in the shedding of old cuticle in a phenomenon called "ecdysis" or "moulting". Nerolidol might exhibit serious disturbances during larval moulting, indicating disruption of the function of larval endocrine system, thereby preventing completion of moulting [151]. For some detail, nerolidol might suppress the activity of ecdysone in larvae leading to the failure of moult and ultimately died $[156,157,158,159,160]$. On the other hand, failure of ecdysis of $S$. littoralis larvae, in the current study, may be attributed to an inhibitory effect of nerolidol on the chitin formation $[6,111]$ or to the inability of larvae to shed their exocuticle during ecdysis [112].

\section{Production of intermediate creatures}

Another feature of disrupted developmental program in insects is the production of larval-pupal or/and pupal-adult intermediates. The formation of some intermediates had been reported for different insect species as response to the disruptive effects of some botanicals [161, 162], such as Tribolium confusum after treatment of $5^{\text {th }}$ instar larvae (production of larval-pupal intermediates) or $6^{\text {th }}$ instar larvae or 0-hr-old pupae (production of pupal-adult intermediates) with $1 \mu \mathrm{g} / \mu \mathrm{l}$ of Andrographolide (a terpenoid) [163]. Also, larval treatment of S. litura with the same plant compound led to the production of larval-pupal intermediates, at all concentrations [164]. Recently, Ghoneim et al. [108] observed some larval-pupal intermediates after treatment of $5^{\text {th }}$ instar or $6^{\text {th }}$ instar larvae with Farnesol, in a dose-dependent course. Results of the present study on $S$. littoralis were, to a great extent, agreed with these reported results, since the treatment of $5^{\text {th }}$ instar or $6^{\text {th }}$ instar larvae of $S$. littoralis with nerolidol led to the production of some larval-pupal intermediates increasingly with the increasing concentration. Irrespective of the larval instar under treatment, the important features of these intermediates had been observed with pupal abdomen and larval head and thorax.

To explicate the production of larval-pupal intermediates in S. littoralis by nerolidol, in the present study, this sesquiterpene compound might interfere with the pupal moulting and development via the disturbance of hormonal regulation, such as the moulting hormone, leading to an ecdysteroid reduction [163]. However, it may be important to provide some suggestions for explicating this criterion of disrupted development program. (1) Nerolidol might inhibit the development program via the interference with the release of the neurosecretion [165]. (2) The production of these intermediates might indicate a juvenile hormone-like activity of nerolidol retarding the perfect larval-pupal transformation. (3) Nerolidol might interfere with the chitin biosynthesis and chitin synthase leading to moulting into non-viable forms between stages [166]. (4) The production of these mosaic creatures in S. littoralis may be explicated by an inhibitory effect of nerolidol on the DNA synthesis. (5) The moult induction had lethal consequences because the induction of a rapid moult did not provide enough time for the completion of larval-pupal transformation. Thus, the insects moulted to non-viable forms between the stages [166]. Molts had been induced during the early phase of the last instar to produce larval-like individuals, while those formed in the late phase generate pupal-like individuals [167]. (6) Nerolidol might cause a misexpression of $b r-\mathrm{C}$ which then leads to improper expression of one or more downstream effector genes controlled by $b r$-C gene products. Symptoms of the impaired development, like larval-pupal intermediates, are the end results [168, 169].

\section{Impaired metamorphosis of $S$. littoralis by nerolidol Inhibited pupation}

As reported by some studies, pupation rate of different insects was suppressed after treatment with plant extracts or plant-derived compounds [144, 148, 170]. In the present study, nerolidol exerted a strong inhibitory action on pupation, since pupation rate was regressed in a dose-dependent course, after treatment of $5^{\text {th }}$ instar or $6^{\text {th }}$ instar larvae. The pupation impediment increased proportional to the increasing nerolidol concentration. This result was in accordance with some reported results of inhibited pupation of some insects 
Ghoneim K et al., Sch Acad J Biosci, Feb, 2021; 9(2): 36-57

after treatment with various plant products. For examples, treatment of the $S$. frugiperda larvae with doses $0.2-5.0 \mu \mathrm{g} / \mathrm{mL}$ of eucalyptin, chrysin, eucalyptin, quercetin, luteolin, and betulinic and oleanolic acids considerably reduced the pupation [171]. Addition of alantolactone and isoalantolactone to the diet of $3^{\text {rd }}$ instar larvae of $S$. litura significantly reduced the pupation $\%$ [144]. A reduction of pupation was recorded in $S$. litura after larval feeding on Miraculan-treated diet [140]. Also, reduction of pupation was observed in $G$. mellonella after injection of ABA into the larval haemocoel [149]. After treatment of $5^{\text {th }}$ or $6^{\text {th }}$ instar larvae of $S$. littoralis with Farnesol, the pupation rate was drastically suppressed [108].

To understand the regressed pupation rate of $S$. littoralis, in the current investigation, nerolidol might exert a suppressive action on the chitin synthesis and prevented the normal deposition of new cuticle during apolysis [172]. For some detail, nerolidol might exert an inhibitory action on the prothoracic gland (ecdysone-producing gland) and hence the ecdysone could not be synthesized and/or released. In other words, nerolidol might block the release of morphogenic peptides, causing disturbance in titers of both ecdysteroids and juvenoids [173]. Also, nerolidol might disrupt the ecdysteroid metabolism or might alternatively act directly to inhibit the release of ecdysis-triggering hormone [174]. In addition, reduction of the pupation rate of $S$. littoralis might be due to inhibitory effect of nerolidol on the synthesis of specific storage proteins in fat body during the last larval instar and their deposition at the time of pupation [175].

\section{Blocked adult emergence}

It is known from the literature sources that the adult emergence of different insects was completely or partially blocked by various plant extracts $[170,156$, 176-180, 144, 181-183]. In the present study, nerolidol appeared to have a weak blocking potency on the adult emergence because partially blocked adult emergence was recorded only at the higher concentrations of Nerolidol, irrespective of the larval instar under treatment. The present result was in agreement with many reported results of significantly blocked adult emergence after larval treatment with some plant products or compounds, such as $S$. littoralis after treatment of $2^{\text {nd }}$ instar larvae of $S$. littoralis with 5,6-dihydroxy-3,4-7 trimethoxy flavones [91] and after treatment of $5^{\text {th }}$ or $6^{\text {th }}$ instar larvae with Farnesol (Sesquiterpene compound) [108].

Apart from S. littoralis, significantly blocked adult emergence of $S$. frugiperda was recorded by Céspedes et al. [138] after treatment of the neonate larvae with gedunin, photogedunin epimeric mixture, photogedunin acetates mixture or Toosendanin, and Salazar et al. [171] after treatment with eucalyptin, chrysin, eucalyptin, and quercetin, luteolin, and oleanolic acids. Also, partially blocked adult emergence of $T$. confusum was observed after treatment of $5^{\text {th }}$ or $6^{\text {th }}$ instar larvae with Andrographolide (a terpenoid) [163]. The adult emergence of $S$. litura and $S$. exigua had been blocked after larval treatment with Pogostone [95]. Blocked adult emergence of $H$. armigera and $S$. litura had been reported after treatment larvae with Flindersine (an alkaloid) [184]. Also, blocked adult emergence of $S$. litura was recorded after feeding of $2^{\text {nd }}$ instar larvae on fresh food treated with Allyl isothiocyanate (an isothiocyanate) [180] or after treatment of $3^{\text {rd }}$ instar larvae with alantolactone and isoalantolactone (sesquiterpenes) [144]. Djeghader et al. [150] reported a blocked adult emergence of $C$. pipiens after treatment of $4^{\text {th }}$ instar larvae with Saponin. In addition, a similar result on B. cucurbitae was recorded by Kaur and Rup [139] after treatment the larvae with the plant growth regulators, $\mathrm{Cn}$, kinetin, $\mathrm{GA}_{3}$ and IAA. Among the pure compounds (isolated from Warburgia ugandensis extracts), polygodial and ugandensolide exhibited significantly higher blocking effects on adult emergence of the larger grain borer Prostephanus truncates [109].

Prior to the interpretation of blocked adult emergence of $S$. littoralis, in the present study, it may be important to mention that the adult emergence is a prerequisite process of the insect metamorphosis. This crucial physiological process has been regulated by the eclosion hormone. Disturbance of this hormone partially or completely arrest the adults to emerge [165]. For interpretation of the blocking of adult emergence after treatment of $5^{\text {th }}$ or $6^{\text {th }}$ instar larvae of S. littoralis, in the present study, nerolidol might exhibit a disturbing effect on the normal metabolism of insect hormones during the development of the immatures leading to failure of adult emergence. In particular, nerolidol might disturb the adult eclosion hormone release and/or inhibition of the neurosecretion [165, 185]. On the molecular basis, nerolidol might cause misexpression of certain genes, particularly the broodcomplex $(b r-C)$ transcription factor gene, leading to symptoms of impaired metamorphosis, like blocking of adult emergence [168, 169].

\section{Perturbed morphogenesis program of $S$. littoralis by nerolidol}

As reported in the current literature, plant extracts of different families or isolated plant compounds drastically affect the morphogenesis of pupae in several insects, as appeared in pupal deformities $[158,163,186$, $151,171,180,160]$. In the present study, nerolidol failed to exert anti-morphogenic action on S. littoralis after treatment of $5^{\text {th }}$ instar larvae. On the other hand, treatment of $6^{\text {th }}$ instar larvae with the higher two concentrations of nerolidol impaired the morphogenesis of some pupae. The malformed pupae developed with bent abdomens, hump-backs or with last larval exuvia attached to head and mouth parts. The present result was in corroboration with some reported results of 
Ghoneim K et al., Sch Acad J Biosci, Feb, 2021; 9(2): 36-57

malformed pupae of different insects after treatment with various plant compounds, such as $S$. littoralis after treatment of $5^{\text {th }}$ or $6^{\text {th }}$ instar larvae of with Farnesol [108]; S. frugiperda after treatment of larvae with eucalyptin, chrysin, eucalyptin, quercetin, luteolin, and oleanolic acids [171] and S. litura after treatment of larvae with Andrographolide [164].

To explicate the disruption of pupation program in S. littoralis, in the present study, nerolidol might inhibit the chitin synthesis or/and might prevent the normal deposition of new cuticle during apolysis leading to the pupal deformities [172]. The anti-morphogenic effect of nerolidol might be due to the disturbance of release of ecdysteroids responsible for the form of developing pupae [138]. In this regard, nerolidol might block the release of morphogenic peptides, causing alteration in titers of juvenoids required for the perfect pupal transformation [173].

\section{CONCLUSION}

Depending on results of the present study, nerolidol exhibited considerably toxic effect on $S$. littoralis, caused serious reduction of larval weight gain and detrimentally inhibited growth and development; disturbed development program, remarkably suppressed pupation, partially blocked adult emergence, and deformed pupae. Therefore, nerolidol could be recommended as an eco-friendly alternative to synthetic insecticides for the management of this dangerous pest.

\section{REFERENCES}

1. Cai Y, Xie Y, Li J. Glandless seed and glanded plant research in cotton: a review. Agronomy for Sustainable Development. 2010; 30: 181-190.

2. Bertrand JA, Sudduth TQ, Condon A, Jenkins TC, Calhoun MC. Nutrient content of whole cottonseed. Journal of Dairy Science. 2005; 88:1470-1477.

3. Hagenbucher S, Eisenring M, Meissle M, Rathore KS, Romeis J. Constitutive and induced insect resistance in RNAi-mediated ultra-low gossypol cottonseed cotton. BMC Plant Biology, 2019; 19: 322 , https://doi.org/10.1186/s12870-019-1921-9

$10 \mathrm{pp}$.

4. Smagghe G, Degheele D. Comparative toxicity and tolerance for the ecdysteroid mimic tebufenozide in a laboratory strain of the cotton leafworm, Spodoptera littoralis (Boisd.)(Lepidoptera: Noctuidae). Journal of Economic Entomology. 1997; 90:278-282.

5. El-Aswad AF, Abdelgaleil SA, Nakatami M. Feeding deterrent and growth inhibitory properties of limonoids from Khaya senegalensis against the cotton leafworm, Spodoptera littoralis. Pest Management Science. 2003; 60: 199-203.

6. Abdel-Rahman SM, Hegazy EM, Elwey AE. Direct and latent effects of two chitin synthetic inhibitors to Spodoptera littoralis (Biosd.) larvae.
American-Euraslian Journal of Agriculture \& Environmental Sciences. 2007; 2(4): 457- 464.

7. Shalaby MM, El-Sherif AG, Borham SG, El-Bialy FM. Toxicity of some essential plant oils against cotton leaf worm, Spodoptera littoralis (Boisd.). Egyptian Academic Journal of Biological Sciences (F. Toxicology \& Pest Control). 2020; 12(1): 01-07.

8. Pineda S, Chneider MS, Smagghe G, Martinez A, Stal PD, Vinuela E, Valle J, Budia F. Lethal and sublethal effects of methoxyfenozide and spinosad on Spodoptera littoralis (Lepidoptera: Noctuidae). Journal of Economic Entomology. 2007; 100: 773-780.

9. Lanzoni A, Bazzocchi GG, Reggiori F, Rama F, Sannino L, Maini S, Burgio G. Spodoptera littoralis male capture suppression in processing spinach using two kinds of synthetic sex-pheromone dispensers. Bulletin of Insectology. 2012; 65(2): 311-318.

10. EPPO. Spodoptera littoralis distribution. EPPO Global Database. 2019; Available: https://gd.eppo.int/taxon/SPODLI/distribution [5 February 2019]

11. El-Aswad AF. Efficiency of certain insecticides and insect growth regulators alone or in mixture with chlorpyrifos for the integrated control of the Egyptian cotton leafworm. Journal of Pest Control and Environmental Science. 2007; 15(2): 29-48.

12. El-Sabrout A. Effects of some materials from plant origin on the cotton leafworm, Spodoptera littoralis. Ph.D.Thesis, Alexandria University, Faculty of Agriculture, Egypt, 2013.

13. Azzouz H, Kebaili-Ghribi J, Ben Farhat-Touzri D, Daoud F, Fakhfakh I, Tounsi S, Jaoua S. Selection and characterisation of an HD1-like Bacillus thuringiensis isolate with a high insecticidal activity against Spodoptera littoralis (Lepidoptera: Noctuidae). Pest Management Science. 2014; 70(8): 1192-1201. doi: 10.1002/ ps.3661.

14. Kandil MA, Abdel-Aziz NF, Sammour EA. Comparative toxicity of chlofluazuron and lufenuron against cotton leafworm, Spodoptera littoralis. Egyptian Journal of Agricultural Research, National Research Center. 2003; 2: 645-661.

15. Bakr RFA, Abd Elaziz MF, El-barky NM, Awad MH, Abd El-Halim HME. The activity of some detoxification enzymes in Spodoptera littoralis (Biosd.) larvae (Lepidoptera-Noctuidae) treated with two different insect growth regulators. Egyptian Academic Journal of Biological Sciences. 2013; 5(2):19-27.

16. Benelli G, Canale A, Toniolo C, Higuchi A, Murugan K, Pavela R. Neem (Azadirachta indica): Towards the ideal insecticide? Natural Product Research. 2017; 31(4): 369-386.

17. El-Sinary NH, Ashour AT, Megahed FA. Water extracts from leaves of Morus albavarieties as botanical pesticides against the cotton leafworm, 
Ghoneim K et al., Sch Acad J Biosci, Feb, 2021; 9(2): 36-57

Spodoptera littoralis (Boisd.). Bulletin of Entomological Society of Egypt (Econ. Ser.). 2008; 34: 69-79.

18. El-Zoghby FA, Salem MH, Gadelhak GG and El-Sabrout AM. Effects of Melilotus indica crude extracts and cascade (IGR) on Spodoptera littoralis (Lepidoptera: Noctuidae) reproductive organs. Bulletin of Entomological Society of Egypt (Econ. Ser.). 2011; 37; 121-136.

19. Sut S, Pavela R, Kolarčik V, Lupidi G, Maggi F, Dall'Acqua S, Benelli G. Isobutyrylshikonin and isovalerylshikonin from the roots of Onosma visianii inhibit larval growth of the tobacco cutworm Spodoptera littoralis. Industrial Crops and Products. 2017; 109: 266-273.

20. Al-Nagar NMA, Abou-Taleb HK, Shawir MS, Abdelgaleil SAM. Comparative toxicity, growth inhibitory and biochemical effects of terpenes and phenylpropenes on Spodoptera littoralis (Boisd.). Journal of Asia-Pacific Entomology. 2020; 23: 67-75.

21. Temerak SA. Historical records of cotton leafworm (Spodoptera littoralis) resistance to conventional insecticides as influenced by the resistance programs in Egypt from 1950-2002. Resistant Pest Management. 2002; 12: 33-36.

22. Abd El-Mageed AEM, Shalaby SEM. Toxicity and bio-chemical impacts of some new insecticide mixtures on cotton leafworm Spodoptera littoralis (Boisd.). Plant Protection Science. 2011; 47(4): 166-175.

23. Ghoneim K, Hamadah Kh, El-Hela A. Acetylcholinesterase activity in the desert Locust Schistocerca gregaria (Acrididae) (Forsk.) as a response to the action of the wild herb Fagonia bruguieri DC. (Zygophyllaceae) extracts. Journal of Entomological Research Society. 2012; 14(2): 87-97.

24. Fetoh BA, Mohamed SA, Seleman LEM. Field and semi field applications for bio and chemical pesticides on cotton leaf worm, Spodoptera littoralis (Boisd.) (Lepidoptera: Noctuidae). Journal of Plant Protection and Pathology, Mansoura University (Egypt). 2015; 6(11): 1471-1478.

25. Aydin MH, Gurkan MO. The efficacy of spinosad on different strains of Spodoptera littoralis (Boisduval) (Lepidoptera: Noctuidae). Turkish Journal of Biology. 2006; 30: 5-9.

26. Mosallanejad H, Smagghe G. Biochemical mechanisms of methoxyfenozide resistance in the cotton leafworm Spodoptera littoralis. Pest Management Science. 2009; 65: 732-736.

27. Rizk GA, Hashem HF, Mohamed SA. Plants in pest control. 2. Evaluation of some plant extracts against the cotton leaf worm, Spodoptera littoralis (Boisd.). Bulletin of Entomological Society of Egypt (Econ. Ser.), 2010; 36: 213-222.

28. Zahran HA, Abdelgaleil SAM. Insecticidal and developmental inhibitory properties of monoterpenes on Culex pipiens L. (Diptera: Culicidae). Journal of Asia-Pacific Entomology. 2011; 14: 46-51. https://doi.org/10.1016/j.aspen. 2010.11.013

29. Metayi MHA, Shekeban MMK, El-Deeb AS. Evaluation of three semi-artificial diets for cotton leafworm mass rearing and their effects on some biological parameters. Alexandria Journal of Agricultural Sciences. 2016; 61(3): 237-241.

30. Eldesouky SE, Khamis WM, Hassan SM. Joint action of certain fatty acids with selected insecticides against cotton leafworm, Spodoptera littoralis and their effects on biological aspects. Journal of Basic and Environmental Sciences. 2019; 6: 23-32.

31. El-Zemaity MS, El-Deeb WM, Osman YA, Hussien AI. Development of resistance of Spodoptera littoralis to certain bioinsecticides. Journal of Environmental Science, 2003; 6: 793-810.

32. Pavunraj M, Baskar K, Paulkumar K, Janarthanan S, Rajendran P. Antifeedant activity of crude extracts and fractions isolated from Catharanthus roseus leaf against spotted bollworm, Earias vittella. Phytoparasitica. 2016; 44: 419-422.

33. Sanni NB, Mutta SR. Impact of solvents leading to environmental pollution. Journal of Chemical and Pharmaceutical Sciences. 2014; 3: 50-52.

34. Damala CA. Potential uses of turmeric ('Curcuma longa') products as alternative means of pest management in crop production. Plant Omics. 2011; 4: 136-141.

35. Korrat EEE, Abdelmonem AE, Helalia AAR, Khalifa HMS. Toxicological study of some conventional and nonconventional insecticides and their mixtures against cotton leaf worm, Spodoptera littoralis (Boisd.) (Lepidoptera: Noctuidae). Annals of Agricultural Science. 2012; 57: 145-152.

36. Gill HK, Garg H. Pesticides: environmental impacts and management strategies. In: "Pesticides-Toxic Aspects". InTech. 2014.

37. Isman MB, Akhtar Y. Plant natural products as a source for developing environmentally acceptable insecticides. In: "Insecticides Design Using Advanced Technologies" (Shaaya I. Nauen R, Horowitz AR., eds.). Springer, Berlin, Germany. 2007: 235-248.

38. Zahoor AMK, Zahoor MA, Mubarik MS, Rizvi H, Majeed HN, Zulhussnain M, Ranian K, Sultan K, Imran M, Qamer S. Insecticidal, biological and biochemical response of Musca domestica (Diptera: Muscidae) to some indigenous weed plant extracts. Saudi Journal of Biological Sciences. 2020; 27(1):106-116.

39. Miresmailli S, Isman MB. Botanical insecticides inspired by plant-herbivore chemical interactions. Trends Plant Science. 2014; 19: 29-35.

40. Pavela R. History, presence and perspective of using plant extracts as commercial botanical insecticides and farm products for protection against insects - a 
Ghoneim K et al., Sch Acad J Biosci, Feb, 2021; 9(2): 36-57

review. Plant Protection Science. 2016; 52: 229241.

41. Szczepanik M, Gliszczynska A, Hnatejko M, Zawitowska B. Effects of halolactones with strong feeding-deterrent activity on the growth and development of larvae of the lesser mealworm, Alphitobius diaperinus (Coleoptera: Tenebrionidae). Applied Entomology and Zoology, 2016; 51:393401.

42. Hernández-Carlos B, Gamboa-Angulo M. Insecticidal and nematicidal contributions of Mexican flora in the search for safer biopesticides. Molecules, 2019; 24: 897-940. https://doi.org/10.3390/ molecules24050897

43. Bullangpoti V, Wajnberg E, Audant P, Feyereisen R. Antifeedant activity of Jatropha gossypifolia and Melia azedarach senescent leaf extracts on Spodoptera frugiperda (Lepidoptera: Noctuidae) and their potential use as synergists. Pest Management Science. 2012; 68(9): 1255-1264.

44. Lakshmanan S, Thushimenan S, Tamizhazhagan V. Antifeedant, larvicidal and oviposition detergent activity of Pongamia pinnata and Ceiba pentandra against pod borer larvae of Helicoverpa armigera (Noctuidae: Lepidoptera). Indo-American Journal of Pharmaceutical Sciences. 2017; 4(2): 180-185.

45. Abdelgaleil SAM, Abou-Taleb HK, Al-Nagar NMA, Shawir MS. Antifeedant, growth regulatory and biochemical effects of terpenes and phenylpropenes on Spodoptera littoralis Boisduval. International Journal of Tropical Insect Science. 2020; 40: 423-433.

46. Abdelgaleil SAM, Mohamed MIE, Badawy MEI, El-Arami SAA. Fumigant and contact toxicities of Monoterpenes to Sitophilus oryzae (L.) and Tribolium castaneum (Herbst) and their inhibitory effects on Acetylcholinesterase activity. Journal of Chemical Ecology, 2009; 35: 518-525..

47. Wu H, Wu H, Wang W, Liu T, Qia M, Feng J, Li X, Liu Y. Insecticidal activity of sesquiterpene lactones and monoterpenoid from the fruits of Carpesiuma brotanoides. Industrial Crops and Products, 2016; 92: 77-83.

48. Saad MG, Abou-Taleb HK, Abdelgaleil SAM. Insecticidal activity of monoterpenes and phenylpropenes against Sitophilus oryzae L. and their acetylcholinesterase and adenosine triphosphatases inhibitory effects. Applied Entomology and Zoology, 2018; 53:173-181.

49. Zahran HA, Abdelgaleil SAM. Insecticidal and developmental inhibitory properties of monoterpenes on Culex pipiens L. (Diptera: Culicidae). Journal of Asia-Pacific Entomology. 2011; 14: 46-51. https://doi.org/10.1016/j.aspen. 2010.11 .013

50. Rajkumar V, Gunasekaran C, Christy IK, Dharmaraj J, Chinnaraj P, Paul CA. Toxicity, antifeedant and biochemical efficacy of Mentha piperita L. essential oil and their major constituents against stored grain pest. Pesticide Biochemistry and Physiology. 2019; 156: 138-144. https://doi.org/10.1016/j.pestbp. 2019.02.016

51. Watanabe Y, Mihara R, Mitsunaga T, Yoshimura T. Termite repellent sesquiterpenoids from Callitris glaucophylla heartwood. Forest Ecology and Management. 2005; 258: 1918-1923.

52. Peixoto MG, Bacci L, Blank AF, Araújo APA, Alves PB, Silva JHS, Santos AA, Oliveira AP, da Costa AS, Arrigoni-Blank MF. Toxicity and repellency of essential oils of Lippia alba chemotypes and their major monoterpenes against stored grain insects. Industrial Crops and Products. 2015; 71: 31-36. https://doi. org/10.1016/j.indcrop.2015.03.084

53. Gonzalez AG, Jimenez IA, Ravelo AG, Coll J, Gonzalez JA, Lloria J. Antifeedant activity of sesquiterpene from celastraceae. Biochemical Systematics and Ecology. 1997; 25: 513-519.

54. Zapata N, Budia F, Vinuela E, Medina P. Antifeedant and growth inhibitory effects of extracts and drimanes of Drimys winteristem bark against Spodoptera littoralis (Lep., Noctuidae). Industrial Crops and Protects. 2009; 30: 119-125. DOI: 10.1016/ j.indcrop. 2009.02.009

55. Ali AM, Mohamed DS, Shaurub EH, Elsayed AM. Antifeedant activity and some biochemical effects of garlic and lemon essential oils on Spodoptera littoralis (Boisduval)(Lepidoptera: Noctuidae). Journal of Entomology and Zoology Studies. 2017; 5: 1476-1482.

56. Abdelgaleil SAM, Abbassy MA, Belal AH, Abdel-Rasoul MAA. Bioactivity of two major constituents isolated from Artemisia judaica L. Bioresource Technology. 2008; 99: 5947-5950. DOI: 10.1016/j.biortech.2007.10.043

57. Abbassy MA, Abdelgaleil SAM, Rabie RYA. Insecticidal and synergistic effects of Majorana hortensis essential oil and some of its major constituents. Entomologia Exprimentalis et Applicata. 2009; 131: 225-232. https://doi.org/ 10.1111/j.1570-7458.2009. 00854.x

58. Abdelgaleil SAM. Molluscicidal and insecticidal potential of monoterpenes on the white garden snail, Theba pisana (Muller) and the cotton leafworm, Spodoptera littoralis (Boisduval). Applied Entomology and Zoology. 2010; 45: 425-433. https://doi.org/10.1303/ aez.2010.425

59. Chan W-K, Tan LT-H, Chan K-G, Lee L-H, Goh B-H. Nerolidol: A sesquiterpene alcohol with multi-faceted pharmacological and biological activities. Molecules. 2016; 21(5): 529.

60. Chen Sh, Zhang L, Cai X, Li X, Bian L, Luo Z, Li Z, Chen Z, Xin Z. (E)-Nerolidol is a volatile signal that induces defenses against insects and pathogens in tea plants. Horticulture Research, 2020; 7:52, 15pp. https://doi.org/10.1038/ s41438-020-0275-7

61. Boncan DAT, Tsang SSK, Li C, Lee IHT, Lam H-M, Chan T-F, Hui JHL. Terpenes and Terpenoids 
Ghoneim K et al., Sch Acad J Biosci, Feb, 2021; 9(2): 36-57

in Plants: Interactions with Environment and Insects. International Journal of Molecular Sciences. 2020; 21, 7382, 19.

62. Wroblewska-Kurdyk A, Dancewicz K, Gliszczynska A, Gabrys B. New insight into the behavior modifying activity of two natural sesquiterpenoids farnesol and nerolidol towards Myzus persicae (Sulzer) (Homoptera: Aphididae). Bulletin of Entomological Research. 2020; 110: 249-258.

63. Favaris AP, Túler AC, Silva WD, Rodrigues SR, Leal WS Bento JMS. (3S,6E)-nerolidol-mediated rendezvous of Cyclocephala paraguayensis beetles in bottle gourd flowers. PLoS ONE. 2020; 15(12): $\mathrm{e} 0235028$.

64. Koudou J, Abena AA, Ngaissona P, Bessiére JM. Chemical composition and pharmacological activity of essential oil of Canarium schweinfurthii. Fitoterapia. 2005; 76: 700-703.

65. Lapczynski A, Bhatia SP, Letizia CS, Api AM. Fragrance material review on farnesol. Food and Chemical Toxicology. 2008; 46: 149-156. https://doi.org/ 10.1016/j.fct.2008.06.046

66. Arruda DC, D’Alexandri FL, Katzin AM, Uliana SRB. Antileishmanial activity of the terpene Nerolidol. Antimicrobial agents and Chemotherapy. 2005; 49(5): 1679-1687.

67. Klopell FC, Lemos M, Sousa JP, Comunello E, Maistro EL, Bastos JK. Nerolidol, an antiulcer constituent from the essential oil of Baccharis dracunculifolia DC. (Asteraceae). Zeitschrift fur Naturforschung. C, Journal of biosciences. 2007; 62: 537-542.

68. Nogueira Neto JD, Cardoso de Almeida AA, Silva Oliveira J, Santos PS, Pergentino de Sousa D, Mendes de Freitas R. Antioxidant effects of nerolidol in mice hippocampus after open field test. Neurochemical Research. 2013; 38: 1861-1870.

69. Javed M, Majeed MZ, Arshad M, Ahmad MH, Abdul Ghafoor H. Insecticidal potentiality of Eruca sativa (mill.), Piper nigrum (1.) and Withania somnifera (1.) extracts against Trogoderma granarium (everts) (Coleoptera: Dermestidae). International Journal of Fauna and Biological Studies. 2016; 3(1): 18-20.

70. Binder BF, Robbins JC, Wilson RL. Chemically mediated ovipositional behaviors of the European corn borer, Ostrinia nubilalis (Lepidoptera: Pyralidae). Journal of Chemical Ecology. 1995; 21, 1315-1327. doi: 10.1007/BF02027564.

71. Wheeler GS, Massey LM, Southwell IA. Antipredator defense of biological control agent Oxyops vitiosais mediated by plant volatiles sequestered from the host plant Melaleuca quinquenervia. Journal of Chemical Ecology. 2002; 28: 297-315.

72. Chantraine J- M, Laurent D, Ballivian C, Saavedra G, Ibañez R, Vilaseca LA. Insecticidal activity of essential oils on Aedes aegypti larvae. Phytotherapy
Research. 1998; 12(5): 350-354. https://doi.org/10.1002/(SICI)1099-1573

(199808)12:5<350::AID-PTR311>3.0.CO;2-7

73. Priestley CM, Burges IF, Williamson EM. Lethality of essential oil constituents towards the human louse, Pediculus humanus, and its eggs. Fitoterapia. 2006; 77: 303-309. DOI: 10.1016/j. fitote.2006.04.005

74. Di Campli E, Di Bartolomeo S, Delli Pizzi P, Di Giulio M, Grande R, Nostro A, Cellini L. Activity of tea tree oil and nerolidol alone or in combination against Pediculus capitis (head lice) and its eggs. Parasitology Research. 2012; 111:1985-1992. DOI: 10.1007/ s00436-012-3045-0

75. Wróblewska-Kurdyk A, Ewa KD, Gliszczyńska A, Gabrys B. New insight into the behaviour modifying activity of two natural sesquiterpenoids farnesol and nerolidol towards Myzus persicae (Sulzer) (Homoptera: Aphididae). Bulletin of Entomological Research. 2019; 110(1):258-249. DOI:10.1017/ S0007485319000609

76. Benelli G, Pavela R, Drenaggi E, Desneux N, Maggi F. Phytol, (E)-nerolidol and spathulenol from Stevia rebaudiana leaf essential oil as effective and eco-friendly botanical insecticides against Metopolophium dirhodum. Industrial Crops and Products. 2020; 155(1): $112844 . \quad$ DOI: 10.1016/j.indcrop.2020.112844

77. da Silva MMC, da Camara, CAG, de Moraes MM, de Melo JPR, dos Santos RB, Neves RCS. Insecticidal and acaricidal activity of essential oils rich in (E)-Nerolidol from Melaleuca leucadendra occurring in the State of Pernambuco (Brazil) and effects on two important agricultural pests. Journal of the Brazilian Chemical Society. 2020; 31(4): 814-820. https://doi.org/10.21577/0103-5053.20190245

78. Hamadah Kh, Ghoneim K, Waheeb H. Impairing effectiveness of Nerolidol, a sesquiterpene compound, on adult performance and reproductive potential of Egyptian cotton leafworm, Spodoptera littoralis (Lepidoptera: Noctuidae). Egyptian Academic Journal of Biological Sciences (Entomology). 2020; 13(2): 97-120.

79. Ghoneim KS. Physiological studies on endocrine and reproductive systems of the cotton leafworm, Spodoptera littoralis. Unpublished PhD. Thesis, Al-Azhar Univ., Cairo, Egypt. 1985.

80. Bakr RFA, El-barky NM, Abd Elaziz MF, Awad MH, Abd El-Halim HME. Effect of Chitin synthesis inhibitors (flufenoxuron) on some biological and biochemical aspects of the cotton leaf worm Spodoptera littoralis Bosid. (Lepidoptera: Noctuidae). Egyptian Academic Journal of Biological Sciences. 2010; 2(2): 43-56.

81. Abbott WS. A method of computing the effectiveness of insecticide. Journal of Economic Entomology. 1925; 18(2): 265-267. 
Ghoneim K et al., Sch Acad J Biosci, Feb, 2021; 9(2): 36-57

82. Finney DJ. Probit analysis. 3rd ed. Cambridge, England: Cambridge University Press. 1971; 318.

83. Waldbauer GP. The consumption and utilization of food by insects. Advances in Insect Physiology. 1968; $\quad 5$ : 229-288. https://doi.org/10.1016/S0065-2806(08)60230-1

84. Dempster C. The population dynamic of Moroccan locust Dociostarus murcocamus in Cyprus. Anti Locust Bulletin. 1957: 27.

85. Richard AG. Cumulative effects of optimum and suboptimum temperatures on insect development. In: "Influence of Temperature on Biological Systems" (Johnson FH. ed.). American Physiological Society. 1957; 15: 35-38.

86. Jimenez-Peydro R, Gimeno-Martos C, Lopez-Ferror J, Serrano- Delgado C, Moreno-Mari J. Effects of the insect growth regulator, cyromazine, on the fecundity, fertility and offspring development of Mediterranean fruit fly, Ceratitis capitata Wied (Diptera, Tephritidae). Journal of Applied Entomology. 1995; 119: 435-438. https://doi.org/10.1111/j.1439-0418.

87. Moroney MJ. Facts from Figures. $3^{\text {rd }}$ ed., Pinguin Book Ltd. Harmondsworth, Middlesex. 1956; 228.

88. GraphPad InStat ${ }^{\circledR}$ Inc.

7825 Fay Avenue, Suite 230 La Jolla, CA 92037

USA 1998; v. 3.01 GraphPad Software. Available online at:

http://www.graphpad.com/scientific-software/ instat/

89. Pavela R. Acute: synergistic and antagonistic effects of some aromatic compounds on the Spodoptera littoralis Boisd. (Lep.: Noctuidae) larvae. Industrial Crops and Products. 2014; 60: 247-258. https://doi.org/10.1016/j.indcrop.2014.06.030

90. Pavela R, Bartolucci F, Desneux N, Lavoir A-V, Canale A, Maggi F, Benelli G. Chemical profiles and insecticidal efficacy of the essential oils from four Thymus taxa growing in central-southern Italy. Industrial Crops and Products. 2019; 138: 111460. https://doi.org/10.1016/j.indcrop.2019.06.023

91. Abdel-Rahim FME, Mohamed EM, Gad MH. Insecticidal activity of three phytochemicals against the Egyptian cotton leafworm, Spodoptera littoralis (Boisd.) (Noctuidae: Lepidoptera). Egyptian Journal of Agricultural Research. 2007; 85(5): 1771-1783.

92. Yousef H, El-Lakwah SF, El Sayed YA. Insecticidal activity of linoleic acid against Spodoptera littoralis (Boisd.). Egyptian Journal of Agricultural Research. 2013; 91(2): 573-580.

93. Sousa RMOF, Rosa JS, Oliveira L, Cunha A, Fernandes-Ferreira M. Activities of Apiaceae essential oils against armyworm, Pseudaletia unipuncta (Lepidoptera: Noctuidae). Journal of Agricultural and Food Chemistry. 2013; 61: 76617672. https://doi.org/10.1021/jf403096d

94. Ennigrou A, Casabianca H, Laarif A, Hanchi B, Hosni K. Maturation-related changes in phytochemicals and biological activities of the Brazilian pepper tree (Schinus terebinthifolius Raddi) fruits. South African Journal of Botany. 2017; 108: 407-415.

95. Huang S-H, Xian J-D, Kong S-Z, Li Y-C, Xie J-H, Lin J, Chen J-N, Wang H-F, Su Z-R. Insecticidal activity of pogostone against Spodoptera litura and Spodoptera exigua (Lepidoptera: Noctuidae). Pest Management Science. 2014; 70: 510-516. doi: 10.1002/ps.3635.

96. Ahadji-Dabla KM, Brunet J-L, Ketoh GK, Apétogbo GY, Glitho IA, Belzunces LP. Larvicidal activity of a natural botanical Biostop Moustiques ${ }^{\circledR}$ and physiological changes induced in susceptible and resistant strains of Anopheles gambiae Giles (Diptera: Culicidae). The Open Entomology Journal. 2015; 9: 12-19.

97. Wu H, Wu H, Wang W, Liu T, Qia M, Feng J, Li X, Liu Y. Insecticidal activity of sesquiterpene lactones and monoterpenoid from the fruits of Carpesiuma brotanoides. Industrial Crops and Products. 2016; 92: 77-83. https://doi.org/10. 1016/j.indcrop. 2016.07.046

98. Rizvi SAH, Ling S, Tian F, Xie F, Zeng X. Toxicity and enzyme inhibition activities of the essential oil and dominant constituents derived from Artemisia absinthium L. against adult Asian citrus psyllid Diaphorina citri Kuwayama (Hemiptera: Psyllidae). Industrial Crops and Products. 2018; 121: 468-475.

99. Sosa A, Diaz M, Salvatore A, Bardon A, Borkosky $\mathrm{S}$, Vera N. Insecticidal effects of Vernonanthura nebularum against two economically important pest insects. Saudi Journal of Biological Sciences, 2019; 26: 881-889. https://doi.org/ 10.1016/j.sjbs 2018.01.005

100.Tang X-T, Ibanez F, Tamborindeguy C. Concanavalin A Toxicity towards potato psyllid and apoptosis induction in midgut cells. Insects. 2020; 11, 243, 12.

101.Eben A, Sporer F, Vogt H, Wetterauer P, Wink M. Search for alternative control strategies of Drosophila suzukii (Diptera: Drosophilidae): laboratory assays using volatile natural plant compounds. Insects. 2020; 11: 0811, 18.

102. Awad HH, Ghazawy NA, Abdel Rahman KM. Impact of Farnesol on the food consumption and utilization, digestive enzymes and fat body proteins of the desert locust Schistocerca gregaria Forskål (Orthoptera: Acrididae). African Entomology. 2013; 21(1): 126-131.

103.Schulz S. Spider pheromones- a structural perspective. Journal of Chemical Ecology. 2013; 39: $1-14$.

104.Harrewijn P, Oosten AM, Piron PGM. Natural terpenoids as messengers: a multidisciplinary study of their production, biological functions, and practical applications. Kluwer Academic Publishers, Dordrecht. 2001: 1-424. 
Ghoneim K et al., Sch Acad J Biosci, Feb, 2021; 9(2): 36-57

105.Tang X, Chen S, Wang L. Isolation and insecticidal activity of farnesol from Stellera chamaejasme. Asian Journal of Chemistry. 2011; 23(3): 1233-1235.

106. Awad HH. Effect of Bacillus thuringiensis and Farnesol on haemocytes response and lysozymal activity of the black cut worm Agrotis ipsilon larvae. Asian Journal of Biological Sciences. 2012; 5(3): 157-170. DOI: 10.3923/ajbs. 2012.157.170

107.Kumar S, Gupta KK. Influence of Farnesol on growth and development of Dysdercus koenigii. $19^{\text {th }}$ International Conference of Entomology, 2017, held at Paris, France, October, 19-20, 2017.

108. Ghoneim K, Hamadah Kh, Waheeb H. Bioefficacy of Farnesol, a common sesquiterpene, on the survival, growth, development, and morphogenesis of Spodoptera littoralis (Lepidoptera: Noctuidae). Egyptian Academic Journal of Biological Sciences (F. Toxicology \& Pest Control). 2020; 12(1): 71-99.

109. Opiyo SA. Evaluation of Warburgia ugandensis Extracts and Compounds for Crop Protection against Prostephanus truncates. Advances in Analytical Chemistry. 2020; 10(2): 15-19.

110.Abdel-Rahman SM, Hegazy EM, Elwey AE. Direct and latent effects of two chitin synthetic inhibitors to Spodoptera littoralis (Biosd.) larvae. American-Euraslian Journal of Agriculture \& Environmental Sciences. 2007; 2(4): 457- 464.

111. Adel MM. Lufenuron impair the chitin synthesis and development of Spodoptera littoralis Boisd. (Lepidoptera: Noctuidae). Journal of Applied Science Research. 2012; 8(5): 2766-2775.

112.Linton YM, Nisbet AJ, Mordue (Luntz) AJ. The effect of azadirachtin on the testes of the desert locust Schistocerca gregaria (Forskal). Journal of Insect Physiology. 1997; 43: 1077-1084. https://doi.org/10.1016/S0022-1910(97) 00060 -7

113. Ghoneim KS, Mohamed HA, Bream SS. Efficacy of the neem seed extract, Neemazal, on growth and development of the Egyptian cotton leafworm, Spodoptera littoralis Boisd. (Lepidoptera: Noctuidae). Journal of Egyptian German Society of Zoology. 2000; 33(E): 161-179.

114.Smagghe G, Degheele D. The significance of pharmacokinetics and metabolism to the biological activity of RH-5992 (tebufenozide) in Spodoptera exempta, Spodoptera exigua and Leptinotarsa decemlineata. Pesticide Biochemistry and Physiology. 1994; 49: 224-234.

115.Osman EE, Rarwash I, El-Samadisi MM. Effect of the anti-moulting agent "Dimilin" on the blood picture and cuticle formation in Spodopterea littoralis (Boisd.) larval. Bulletin of Entomological Society of Egypt (Econ. Ser.). 1984; 14: 3-46.

116.López MD, Pascual-Villalobos MJ. Mode of inhibition of acetylcholinesterase by monoterpenoids and implications for pest control. Industrial Crop Products. 2010; 31: 284-288. DOI:10.1016/J. INDCROP.2009.11.005
117. Yeom H-J, Kang JS, Kim G-H, Park I-K. Insecticidal and acetylcholine esterase inhibition activity of Apiaceae plant essential oils and their constituents against adults of German cockroach (Blattella germanica). Journal of Agricultural and Food Chemistry. 2012; 60: 7194-7203. doi: 10.1021/jf302009w.

118. Chaubey MK. Responses of Tribolium castaneum (Coleoptera: Tenebrionidae) and Sitophilus oryzae (Coleoptera: Curculionidae) against essential oils and pure compounds. Herba Polonica. 2012; 58(3): 33-45. https://doi.org/10.1080/ 01448765. 2012.662792

119. Chaubey MK. Biological effects of essential oils against rice weevil Sitophilus oryzae L. (Coleoptera: Curculionidae). Journal of Essential Oil Bearing Plants. 2012b; 15: 809-815. DOI:10.1080/ 0972060X.2012.10644124

120.Seo S-M, Kim J, Lee S-G, Shin C-H, Shin S-C, Park I-L. Fumigant antitermitic activity of plant essential oils and components from ajowan (Trachyspermum ammi), allspice (Pimenta dioica), caraway (Carum carvi), dill (Anethum graveolens), geranium (Pelargonium graveolens), and litsea (Litsea cubeba) oils against Japanese termite (Reticulitermes speratus Kolbe). Journal of Agricultural and Food Chemistry. 2009; 57: 65966602. doi:10.1021/ jf9015416.

121.Faraone N, Hillier NK, Cutler GC. Plant essential oils synergize and antagonize toxicity of different conventional insecticides against Myzus persicae (Hemiptera: Aphididae). PLoS ONE. 2015; 10(5): e0127774. doi:10.1371/journal.pone. 0127774

122. Kiran S, Prakash B. Assessment of toxicity, antifeedant activity, and biochemical responses in stored-grain insects exposed to lethal and sublethal doses of Gaultheria procumbens L. essential oil. Journal of Agriculture and Food Chemistry. 2015; 63: 10518-10524. DOI: 10.1021/acs.jafc.5b03797

123. Ma Z, Li Y, Wu L, Zhang $\mathrm{X}$. Isolation and insecticidal activity of sesquiterpenes alkaloids from Tripterygium wilfordii Hook. Industrial Crops and Products. 2014; 52: 642-648. https://doi.org/10.1016/j.indcrop.2013. 11.029

124.Herrera JM, Zunino MP, Dambolena JS, Pizzolitto RP, Ganan NA, Lucini EI, Zygadlo JA. Terpene ketones as natural insecticides against Sitophilus zeamais. Industrial Crops and Products. 2015; 70: 435-442. https://doi.org/10.1016/ j.indcrop.2015. 03.074

125.AlShebly MM, AlQahtani FS, Govindarajan M, Gopinath K, Vijayan P, Benelli G. Toxicity of ar-curcumene and epi- $\beta$-bisabolol from Hedychium larsenii (Zingiberaceae) essential oil on malaria, chikungunya and St. Louis encephalitis mosquito vectors. Ecotoxicology and Environmental Safety. 2017; 137: 149-157. doi: 10.1016/j.ecoenv.2016.11.028 
Ghoneim K et al., Sch Acad J Biosci, Feb, 2021; 9(2): 36-57

126.Baranitharan M, Tamizhazhagan V, Kovendan K, Senthilmurugan S. Punica granatum-based green ethanolic extract as highly effective and eco-Friendly larvicide, repellent against medically important mosquito vectors. Entomology and Applied Science Letters. 2019; 6(2): 33-41.

127. Ghoneim K, Hassan HA, Tanani MA, Bakr NA. Toxic and disruptive effects of Novaluron, a chitin synthesis inhibitor, on development of the pink bollworm Pectinophora gossypiella (Saunders) (Lepidoptera: Gelechiidae). International Journal of Entomology Research. 2017; 2(2): 36-47.

128. Talukder FA. Plant products as potential stored-product insect management agents-A mini review. Emirates Journal of Food and Agriculture. 2006; 18(1): 17-32. https://doi.org/10.9755/ejfa

129. Giner M, Avilla J, Balcells M, Caccia S, Smagghe G. Toxicity of allyl esters in insect cell lines and in Spodoptera littoralis larvae. Archives of Insect Biochemistry and Physiology. 2012; 79(1): 18-30. https://doi.org/10.1002/ arch.2012.79.issue-1

130. Abdellaoui K, Ben Halima-Kamel M, Ben Hamouda MH. The antifeeding and repellent properties of gibberellic acid against Asiatic migratory locust Locusta migratoria migratoria. Tunisian Journal of Plant Protection. 2009; 4: 57-66.

131. Awad HH. The effect of natural compounds on the black cutworm Agrotis ipsilon (Hufnagel). Ph.D. thesis, Cairo University, Giza, Egypt, 2001.

132. Szczepanik M, Gliszczynska A, Hnatejko M, Zawitowska B. Effects of halolactones with strong feeding-deterrent activity on the growth and development of larvae of the lesser mealworm, Alphitobius diaperinus (Coleoptera: Tenebrionidae). Applied Entomology and Zoology. 2016; 51:393401. doi:10.1007/ s13355-016-0411-x.

133. Giongo AMM, Vendramim JD, De Freitas SDL, Da Silva MFGF. Growth and nutritional physiology of Spodoptera frugiperda (Lepidoptera: Noctuidae) fed on Meliaceae fractions. Revista Colombiana de Entomología. 2015; 41(1): 33-40.

134. Abdelgaleil SAM, El-Aswad AF. Antifeedant and growth inhibitory effects of tetranortriterpenoids isolated from three meliaceous species on the cotton leafworm, Spodoptera littoralis (Boisd.). Research Journal of Applied Sciences. 2005; 1:234-241.

135. Adel MM, Zaki FN. Biological response of Spodoptera littoralis larvae to feeding on diet mixed with neem product at different concentrations. Archives of Phytopathology and Plant Protection. 2010; 43: 775-782. https://doi.org/10. 1080/03235400802246697

136.Pavela R. Screening of Eurasian plants for insecticidal and growth inhibition activity against Spodoptera littoralis larvae. African Journal of Agricultural Research. 2011; 6: 2895-2907. https://doi.org/10. 5897/AJAR11.046

137.Pavela R, Vrchotova N. Insecticidal effect of furanocoumarins from fruits of Angelica archangelica L. against larvae of Spodoptera littoralis Boisd. Industrial Crops and Products. 2013; 43: 33-39. https://doi.org/10.1016/j.indcrop.2012. 06.044

138. Céspedes CL, Calderón JS, Lina L, Aranda E. Growth inhibitory effects on fall armyworm Spodoptera frugiperda of some limonoids isolated from Cedrela spp. (Meliaceae). Journal of Agriculture and Food Chemistry. 2000; 48(5): 1903-1908. https://doi. org/10.1021/jf990443q

139. Kaur R, Rup PJ. Influence of four plant growth regulators on development of the melon fruit fly, Bactrocera cucurbitae (Coquillett). Insect Science and its Application. 2003; 23(2): 121-125.

140.Bhatnagar S, Kumar A, Karnatak AK. Influence of synthetic plant growth stimulant, Miraculin, on the survival and development of Spodoptera litura (Fab.)(Lepidoptera: Noctuidae). Indian Forester. 2012; 138(12): 1160-1163.

141.Corzo FL, Gilabert M, Alcaide MF, Bardón A. Toxicity of Porella chilensisses qui- and diterpenoids against larvae of the corn pest Spodoptera frugiperda (J.E. Smith) (Lepidotera: Noctuidae). Neotropical Entomology. 2012; 141:414-419.

142.Szołyga B, Gniłka R, Szumny Szczepanik M. Chemical composition and insecticidal activity of Thuja occidentalis and Tanacetum vulgare essential oils against larvae of the lesser mealworm, Alphitobius diaperinus. Entomologia Experimentalis et Applicata. 2014; 151: 1-10. https://doi.org/10.1111/eea.12166

143. Gordy JW, Leonard BR, Blouin D, Davis JA, Stout MJ. Comparative effectiveness of potential elicitors of plant resistance against Spodoptera frugiperda (J. E. Smith) (Lepidoptera: Noctuidae) in four crop plants. PLoS ONE. 2015; 10(9): e0136689. doi:10.1371/journal.pone.0136689

144. Kaur M, Kumar R, Upendrabhai DP, Singh IP, Kaur $\mathrm{S}$. Impact of sesquiterpenes from Inula racemosa (Asteraceae) on growth, development and nutrition of Spodoptera litura (Lepidoptera: Noctuidae). Pest Management Science. 2017; 73(5): 1031-1038. DOI: $10.1002 /$ ps.4429

145. Sosa A, Costa M, Salvatore A, Bardon A, Borkosky $\mathrm{S}$, Vera N. Insecticidal effects of eudesmanes from Pluchea sagittalis (Asteraceae) on Spodoptera frugiperda and Ceratitis capitata. c International Journal of Environment, Agriculture and Biotechnology. 2017; 2(1): 361-369. DOI: 10.22161/ijeab/ 2.1.45

146. Barrett RDH, Agrawal AA. Interactive effects of genotype, environment, and ontogeny on resistance of cucumber (Cucumis sativus) to the generalist herbivore, Spodoptera exigua. Journal of Chemical Ecology. 2004; 30(1): 37-51. DOI: 10.1023/b:joec. $0000013181.50319 .9 \mathrm{~d}$

147.Ladhari A, Laarif A, Omezzine F, Haouala R. Effect of the extracts of the spiderflower, Cleome arabica, 
on feeding and survival of larvae of the cotton leaf-worm, Spodoptera littoralis. Journal of Insect Science. 2013; 13: 61. DOI: 10.1673/031.013.6101

148. Gupta S, Aror, R, Arora S, Sohal SK. Evaluation of insecticidal potential of 4-Methylthiobutyl isothiocyanate on the growth and development of polyphagous pest, Spodoptera litura (Fab.) (Lepidoptera: Noctuidae). International Journal of Entomology Research. 2017; 2(2): 01-05.

149.Er A, Keskin M. Influence of abscisic acid on the biology and hemocytes of the model insect Galleria mellonella (Lepidoptera: Pyralidae). Annals of the Entomological Society of America. 2015; 109(2): 244-251.

150.Djeghader NE, Aïssaoui L, Amira K, Boudjelida H. Toxicity evaluation and effects on the development of a plant extract, the Saponin, on the domestic mosquito, Culex pipiens. International Journal of Mosquito Research. 2018; 5(1): 01-05.

151.Ben Hamouda A, Boussadia O, Khaoula B, Laarif A, Braham M. Studies on insecticidal and deterrent effects of olive leaf extracts on Myzus persicae and Phthorimaea operculella. Journal of Entomology and Zoology Studies. 2015; 3(6): 294-297.

152.Djeghader NEH, Aïssaoui L, Amira K, Boudjelida H. Impact of a chitin synthesis inhibitor, Novaluron, on the development and the reproductive performance of mosquito Culex pipiens. World Applied Science Journal. 2014; 29(7): 954-960.

153. Awad HH, Ghazawy NA. Effects of Farnesol on the ultrastructure of brain and corpora allata, sex hormones and on some oxidative stress parameters in Locusta migratoria (Orthoptera: Acridiidae). African Entomology. 2016; 24(2):502-512. DOI:10.4001/003. 024.0502

154. Tanzubil PB, Mccaffery AR. Effects of azadirachtin and aqueous neem seed extracts on survival, growth and development of the African armyworm, Spodoptera exempta. Crop Protection. 1990; 9: 383-386. https://doi.org/10. 1016/ 0261-2194(90)90012-V

155. Kuwano E, Tanaka Y, Kikuchi M, Eto M. Effects of anti- juvenile hormones and related compounds on development in the larvae of Bombyx mori. Journal of Faculty of Agriculture, Kyushu University. 1988; 33(1,2): 17-28.

156.Baskar K, Kingsley S, Vendan SE, Paulraj MG, Duraipandiyan V, Ignacimuthu S. Antifeedant, larvicidal and pupicidal activities of Atalantia monophylla (L) Correa against Helicoverpa armigera Hubner (Lepidoptera: Noctuidae). Chemosphere. 2009; 75(3): 355-359. https://doi.org/10.1016/j.chemosphere. 2008.12.034

157.Baskar K, Sasikumar S, Muthu C, Kingsley S, Ignacimuthu S. Bioefficacy of Aristolochia tagala Cham. against Spodoptera litura Fab. (Lepidoptera: Noctuidae). Saudi Journal of Biological Sciences. 2011; 18: 23-27. https://doi. org/10.1016/j. sjbs.2010.09.004
158.Jeyasankar A, Elumalai K, Raja N, Ignacimuthu S. Effect of plant chemicals on oviposition deterrent and ovicidal activities against female moth, Spodoptera litura (Fab.) (Lepidoptera: Noctuidae). International Journal of Agricultural Science Research. 2013; 2(6): 206-213.

159. Sivaraman GG, Paulraj M, Ignacimuthu S, Al-Dhabi NA. Bioefficacy of Cleome viscosa L. and Sinapis alba $\mathrm{L}$. seed extracts against Helicoverpa armigera (Hubner) (Lepidoptera: Noctuidae). International Journal of Pure and Applied Zoology. 2014; 2(3): 211-217.

160.Chennaiyan V, Sivakami R, Jeyasankar A. Effect of Duranta erecta Linn (Verbenaceae) leaf extracts against armyworm Spodoptera litura and cotton bollworm Helicoverpa armigera (Lepidoptera: Noctuidae). International Journal of Advanced Research of Biological Sciences. 2016; 3(2): 311-320.

161.Kaur T, Vasudev A, Sohal SK, Manhas RK. Insecticidal and growth inhibitory potential of Streptomyces hydrogenans DH16 on major pest of India, Spodoptera litura (Fab.) (Lepidoptera: Noctuidae). BMC Microbiology. 2014; 14: 227, 9pp.

162.Palanikumar M, Pravin Y, Navaneethan M, Mahendren S, Mohanraj RS, Dhanakkodi B. Callistemon citrinus (Myrtaceae) methanolic leaf extract: a potent mosquitocidal agent for controlling dengue vector mosquito Aedes aegypti (Diptera: Culicidae). Journal of Entomology and Zoology Studies. 2017; 5(3): 1051-1059.

163.Lingampally V, Solanki VR, Kaur A, Raja SS. Andrographolide- an effective insect growth regulator of plant origin against Tribolium confusum (Duval). International Journal of Current Research. 2013; 5(1): 22-26.

164.Edwin E, Vasantha-Srinivasan P, Senthil-Nathan S, Thanigaivel A, Ponsankar A, Selin-Rani S, Kalaivani K, Hunter WB, Duraipandiyan V, Al-Dhabi NA. Effect of andrographolide on phosphatases activity and cytotoxicity against Spodoptera litura. Invertebrate Survival Journal. 2016; 13: 153-163.

165.Josephrajkumar A, Subrahmanyam B, Srinivasan S. Plumbagin and azadirachtin deplete haemolymph ecdysteroid levels and alter the activity profiles of two lysosomal enzymes in the fat body of Helicoverpa armigera (Lepidoptera: Noctuidae). European Journal of Entomology. 1999; 96: 347-353.

166.Tateishi K, Kiuchi M, Takeda S. New cuticle formation and moult inhibition by RH-5849 in the common cutworm, Spodoptera litura (Lepidoptera: Noctuidae). Applied Entomology and Zoology. 1993; 28: 177-184. DOI: https://doi.org/10. 1303/aez.28.177

167.Eizaguirre M, López C, Schafellner Ch, Sehnal F. Effects of ecdysteroid agonist RH-2485 reveal 
Ghoneim K et al., Sch Acad J Biosci, Feb, 2021; 9(2): 36-57

interactions between ecdysteroids and juvenile hormones in the development of Sesamia nonagrioides. Archives of Insect Biochemistry and Physiology. 2007; 65: 74-84.

168. Wilson TG. The molecular site of action of juvenile hormone and juvenile hormone insecticides during metamorphosis: how these compounds kill insects. Journal of Insect Physiology, 2004; 50: 111-121.

169.Nandi PS, Chakravarty K. Juvenoids and anti-Juvenoids as third generation pesticide to control lepidopteran field crop pests. Indian Streams Research Journal. 2011; 1(6): 15.

170.Jilani G, Khattak MK, Shazad F. Toxic and growth regulating effect of ethanol extract and petroleum ether extract of Valariana officianalis against Bactrocera zonata Saunder. Pakistan Entomology. 2006; 28:11-14.

171.Salazar JR, Torres P, Serrato B, Dominguez M, Alarcón J, Céspedes CL. Insect Growth Regulator (IGR) effects of Eucalyptus citriodora Hook (Myrtaceae). Latin American and Caribbean Bulletin of Medicinal and Aromatic Plants. 2015; 14(5): 403-422.

172.Retnakaran A, Granett J, Ennis T. Insect growth regulators. In: "Comprehensive Insect Physiology, Biochemistry, and Pharmacology" (Kerkut G, Gilbert LI. eds.). 12, Pergamon Press, New York. 1985: 529-601.

173.Barnby MA, Klocke JA. Effects of azadirachtin on levels of ecdysteroids and prothoracicotropic hormone-like activity in Heliothis virescens (Fabr.) larvae. Journal of Insect Physiology. 1990; 36: 125-131. https://doi.org/10.1016/0022-1910(90)90183-G

174.Gaur R, Kumar K. Insect growth-regulating effects of Withania somnifera in a polyphagous pest, Spodoptera litura. Phytoparasitica. 2010; 38(3): 237-241. DOI: 10.1007/s12600-010-0092-x

175.Gupta AP. Cellular elements in the haemolymph. In: "Comprehensive Insect Physiology, Biochemistry and Pharmachology"(Kerkt GA, Gilbert LI., eds). Pergamon Press, Oxford. 1985: 401-451.

176. Kaur A, Sohal SK, Singh R, Arora S. Development inhibitory effect of Acacia auriculiformis extracts on Bactrocera cucurbitae (Coquillett) (Diptera: Tephriditae). Journal of Biopesticides. 2010; 3: 499-504.

177.Baskar K, Ignacimuthu S. Antifeedant, larvicidal and growth inhibitory effect of ononitol monohydrate isolated from Cassia tora L. against Helicoverpa armigera (Hub.) and Spodoptera litura (Fab.) (Lepidoptera: Noctuidae). Chemosphere. 2012; 88(4): 384-388. https://doi.org/10.1016/j.chemosphere.2012.02.051

178. Nogueira J, Mourão SC, Dolabela IB, Santos MG, Mello CB, Kelecom A, Mexas R, Feder D, Fernandes CP, Gonzalez MS, Rocha L. Zanthoxylum caribaeum (Rutaceae) essential oil: chemical investigation and biological effects on Rhodnius prolixus nymph. Parasitology Research. 2014; 113: 4271-4279. Doi:10.1007/ s00436-014-4105-4

179.Pathak CS, Tiwari SK. Toxicity of Neem stem bark powder against the ontogeny of rice-moth, Corcyra cephalonica, Staint. (Lepidoptera: Pyralidae). International Journal of Zoological Investigations. 2015; 1(2): 187-191.

180.Bhushan S, Gupta S, Sohal SK, Arora S. Assessment of insecticidal action of 3-Isothiocyanato-1-propene on the growth and development of Spodoptera litura (Fab.) (Lepidoptera: Noctuidae). Journal of Entomology and Zoology Studies. 2016; 4(5): 1068-1073.

181.El-Naggar SEM, Hazaa MAM, Hassan RS, Mehany AL. Insecticidal and growth inhibition activity of some medical plants against cotton leaf worm, Spodoptera littoralis (Boisd). Egyptian Academic Journal of Biological Sciences (F. Toxicology \& Pest control). 2019; 11(2): 58-69.

182.EL-Sabagh MMA, Abd El-Kareem SMI, Abd El Mageed ENI, Amin NS. Comparative between two eco-friendly botanical oils through studies toxicological, biological and molecular impacts on the cotton leafworm, Spodoptera littoralis (Boisd.). Egyptian Academic Journal of Biological Sciences (F. Toxicology \& Pest control). 2019; 11(2): 121-130.

183.Hassan MI, Atwa WA, Moselhy WA, Mahmoud DA. Efficacy of the green tea, Camellia sinensis leaves extract on some biological activities of Culex pipiens and the detection of its phytochemical constituents. Egyptian Academic Journal of Biological Sciences (F. Toxicology \& Pest Control). 2020; 12(1): 59-70.

184.Duraipandiyan V, Baskar K, Muthu Ch, Ignacimuthu S, Al-Dhabi NA. Bioefficacy of Flindersine against Helicoverpa armigera Hübner, Spodoptera litura Fabricius, Anopheles stephensis Liston. and Culex quinquefasciatus Say. Brazilian Archives of Biology and Technology. 2015; 58(4): 595-604.

185.Al-Sharook Z, Balan K, Jiang Y, Rembold H. Insect growth inhibitors from two tropical Meliaceae: Effects of crude seed extracts on mosquito larvae. Journal of Applied Entomology. 1991; 111: 425-430. https://doi.org/10.1111/j.1439-0418. 1991.tb00344.x

186. Scapinello J, de Oliveira JV, Chiaradia LA, Tomazelli Junior O, Niero R, Magro JD. Insecticidal and growth inhibiting action of the supercritical extracts of Melia azedarach on Spodoptera frugiperda. Revista Brasileira de Engenharia Agrícola e Ambiental. 2014; 18(8): 866-872. http://dx.doi.org/10.1590/1807-1929/ agriambi.v18n08p866-872. 